\title{
The first 62 AGN observed with SDSS-IV MaNGA - III: stellar and gas kinematics
}

Gabriele S. Ilha ${ }^{\oplus}{ }^{1,2 \star}$ Rogemar A. Riffel $^{\circledR},{ }^{1,2 \star}$ Jaderson S. Schimoia, ${ }^{1,2,3}$ Thaisa Storchi-Bergmann, ${ }^{2,3}$ Sandro B. Rembold, ${ }^{1,2}$ Rogério Riffel $^{\circledR}, 2,3$ Dominika Wylezalek ${ }^{\oplus},{ }^{4}$ Yong Shi ${ }^{\oplus}, 5$ Luiz N. da Costa, ${ }^{2,6}$ Alice D. Machado,,${ }^{1,2}$ David R. Law, ${ }^{7,8}$ Dmitry Bizyaev, ${ }^{9,10}$ Nicolas D. Mallmann, ${ }^{2,3}$ Janaina Nascimento, ${ }^{2,3}$ Marcio A. G. Maia ${ }^{2,6}$ and Rafael Cirolini ${ }^{1,2}$

${ }^{1}$ Departamento de Física, CCNE, Universidade Federal de Santa Maria, 97105-900, Santa Maria, RS, Brazil

${ }^{2}$ Laboratório Interinstitucional de e-Astronomia - LIneA, Rua Gal. José Cristino 77, Rio de Janeiro, RJ - 20921-400, Brazil

${ }^{3}$ Departamento de Física, IF, Universidade Federal do Rio Grande do Sul, CP 15051, 91501-970, Porto Alegre, RS, Brazil

${ }^{4}$ European Southern Observatory, Karl-Schwarzschildstr 2, D-85748 Garching bei München, Germany

${ }^{5}$ Department of Astronomy, Nanjing University, Nanjing 210093, China

${ }^{6}$ Observatório Nacional - MCT, Rua General José Cristino 77, Rio de Janeiro, RJ - 20921-400, Brazil

${ }^{7}$ Space Telescope Science Institute, 3700 San Martin Drive, Baltimore, MD 21218, USA

${ }^{8}$ Dunlap Institute for Astronomy and Astrophysics, University of Toronto, 50 St George Street, Toronto, Ontario M5S 3H4, Canada

${ }^{9}$ Apache Point Observatory, PO Box 59, Sunspot, NM 88349, USA

${ }^{10}$ Sternberg Astronomical Institute, Moscow State University, 119992 Moscow, Russia

Accepted 2018 December 2. Received 2018 November 21; in original form 2018 May 3

\begin{abstract}
We investigate the effects of active galactic nuclei (AGN) on the gas kinematics of their host galaxies, using MaNGA data for a sample of 62 AGN hosts and 109 control galaxies (inactive galaxies). We compare orientation of the line of nodes (kinematic position angle - PA) measured from the gas and stellar velocity fields for the two samples. We found that AGN hosts and control galaxies display similar kinematic PA offsets between gas and stars. However, we note that AGN have larger fractional velocity dispersion $\sigma$ differences between gas and stars $\left[\sigma_{\text {frac }}=\left(\sigma_{\text {gas }}-\sigma_{\text {stars }}\right) / \sigma_{\text {stars }}\right]$ when compared to their controls, as obtained from the velocity dispersion values of the central (nuclear) pixel (2".5 diameter). The AGN have a median value of $\sigma_{\text {frac }}$ of $\left\langle\sigma_{\text {frac }}>\right.$ AGN $=0.04$, while the median value for the control galaxies is $<\sigma_{\text {frac }}>_{\text {CTR }}=-0.23 .75$ per cent of the AGN show $\sigma_{\text {frac }}>-0.13$, while 75 percent of the normal galaxies show $\sigma_{\text {frac }}<-0.04$, thus we suggest that the parameter $\sigma_{\text {frac }}$ can be used as an indicator of AGN activity. We find a correlation between the [O III] $\lambda 5007$ luminosity and $\sigma_{\text {frac }}$ for our sample. Our main conclusion is that the AGN already observed with MaNGA are not powerful enough to produce important outflows at galactic scales, but at 1-2 kpc scales, AGN feedback signatures are always present on their host galaxies.
\end{abstract}

Key words: galaxies: active-galaxies: general-galaxies: kinematics and dynamics.

\section{INTRODUCTION}

Theoretical studies and numerical simulations suggest that active galactic nuclei (AGN) play an important role in the evolution of their host galaxies (e.g. Hopkins et al. 2005). Currently, it is widely accepted that galaxies with spherical component (bulge of spiral galaxies and elliptical galaxies) host a central supermassive black hole (SMBH; Ferrarese \& Merritt 2000; Gebhardt et al. 2000;

^E-mail: gabrieleilha1994@gmail.com (GSI); rogemar@ufsm.br (RAR)
Tremaine et al. 2002; Scannapieco et al. 2005) and cosmological simulations that do not include feedback effects from the SMBH result in galaxy stellar masses much higher than observed (Di Matteo, Springel \& Hernquist 2005; Springel, Di Matteo \& Hernquist 2005; Bower et al. 2006). Massive outflows originated in the accretion flow are claimed to regulate and couple the growth of the galactic bulge and SMBH (Hopkins et al. 2005) and to explain the relation between the mass of the SMBH and stellar velocity dispersion of the bulge - the $M-\sigma$ relation (e.g. Ferrarese \& Merritt 2000; Gebhardt et al. 2000). 
According to the Unified Model for AGN (e.g. Antonucci 1993; Urry \& Padovani 1995), the narrow-line region (NLR) is expected to present a bi-conical shape, within which gas outflows due to winds from the accretion disc are expected to be observed. However, Hubble Space Telescope (HST) narrow-band [O III] 25007 images of a sample of 60 nearby Seyfert galaxies show that the bi-conical shape of the NLR is not as common as expected (Schmitt et al. 2003), and gas outflows are seen only in 33 per cent of Seyfert galaxies, as revealed by long-slit spectroscopy of 48 nearby AGN (Fischer et al. 2013). Nevertheless, long-slit observations are restricted to only one position angle (PA). A better mapping of the outflows and their geometries can be obtained via integral field spectroscopy (IFS), as shown in recent studies both in the optical and nearinfrared (e.g. Barbosa et al. 2014; Riffel, Storchi-Bergmann \& Riffel 2014; Schnorr-Müller et al. 2014; Lena et al. 2015; Zakamska et al. 2016). The comparison between the gas and stellar kinematics on kiloparsec scales allows the study of the possible impact of AGN outflows on its host galaxy. So far, most studies aimed to investigate gas outflows from AGN have been performed for small samples or individual galaxies. In this work, we use the observations from the Mapping Nearby Galaxies at the Apache Point Observatory (MaNGA) survey (Bundy et al. 2015) to compare the gas and stellar kinematics of a sample composed by 62 AGN observed in the MPL5 (MaNGA Product Launch V) (Data Release 14, Abolfathi et al. 2018) with those of a control sample of inactive galaxies, matched with the AGN sample by properties of the host galaxies. If an AGN sample presents strong outflows, the large-scale gas velocity fields are expected to be disturbed when compared to the stellar velocity fields, while for inactive galaxies, the stellar and gas velocity fields are expected to be similar. Another way that AGN can affect the gas dynamics is by increasing the gas velocity dispersion due to the shocks of the nuclear outflow with the ambient gas.

The AGN and control samples used in this paper are described in Rembold et al. (2017; hereafter Paper I), which presents also the study of the nuclear stellar populations. This is the third paper of a series aimed to compare properties of AGN hosts and their control galaxies. Besides Paper I, the spatially resolved stellar populations are investigated in Mallmann et al. (2018; Paper II). In addition, the gas excitation and distribution will be presented by Nascimento et al. (in preparation - Paper IV).

This paper is organized as follows: Section 2 presents the samples of active and inactive galaxies and the data analysis methods, while Section 3 presents the results, which are discussed in Section 4. Finally, the conclusions of this work are presented in Section 5.

\section{THE DATA AND ANALYSIS}

\subsection{Sample and MaNGA data}

We use the data cubes obtained within the MaNGA survey of the sample of AGN and matched control sample defined in Paper I. The MaNGA survey is part of the fourth-generation Sloan Digital Sky Survey (SDSS-IV) and is aimed to observe $\sim 10000$ nearby galaxies using optical IFS covering the spectral range 3600-10 $000 \AA$ and spectral resolving power $R \sim 2000$ at a spatial resolution of $1-$ $2 \mathrm{kpc}$. The MaNGA sample of galaxies was designed to cover at least $1.5 R_{e}\left(R_{e}-\right.$ effective radius). Here, the effective radius is defined as the radius that contains the half luminosity of galaxy measured at the $i$ band as described in Bundy et al. (2015). The MaNGA survey science goals are presented in Bundy et al. (2015), the design and performance of the integral field units are discussed in Drory et al. (2015), and the MaNGA sample is presented in Wake et al. (2017). Yan et al. (2016b) present the survey design, execution, and data quality, the observing strategy is presented in Law et al. (2015), and the data reduction and calibrations are discussed in Law et al. (2016) and Yan et al. (2016a).

Our sample is composed of the first 62 AGN observed with MaNGA - selected from MaNGA MPL-5 (Data Release 14, Abolfathi et al. 2018). For each AGN, two control inactive galaxies, matched to the AGN hosts in absolute magnitude, galaxy mass, redshift, morphological type and inclination, were selected. The AGN selection realized by Rembold et al. (2017) is based on single-fibre SDSS-III observations. A detailed description and characterization of the AGN and control samples are presented in Paper I. Wylezalek et al. (2018) found 173 galaxies that would not have been selected as AGN candidates based on single-fibre spectral measurements, but MaNGA allowed AGN selection based on the fully spatially resolved optical diagnostics and in the future papers similar work will be done for 'nuclear' AGN and 'off-nuclear' AGN. Thus, in this work we focus on the 'nuclear' AGN. As mentioned in Rembold et al. (2017), our AGN sample includes 34 (55 per cent) spiral and 18 (29 percent) elliptical galaxies. The remaining 10 objects (16 percent) comprise six E/S galaxies, one merger, and three unclassified objects.

\subsection{Spatial filtering and noise removal}

In order to remove noise from the observed data cubes, without loss of angular resolution, we performed a spatial filtering of the data cubes using a Butterworth bandpass filter (Gonzalez \& Woods 2002). This filter is performed in the frequency domain. We used a low-bandpass filter to remove high spatial frequency components from the cubes, which are usually due to spurious features (e.g. bad pixels or cosmic rays). This procedure allows us to improve the fit, emission and absorption line spectra, compared with the original data cubes.

To perform the spatial filtering, we used the Interactive Data Language (IDL) routine bandpass_filter.pro, which allows the choice of the cut-off frequency (v) and order of the filter $n$. A low value of $n$ (e.g. 1) is close to a Gaussian filter, while a high value (e.g. 10) corresponds to an ideal filter. We used $n=5$ and $v=$ $0.25 \mathrm{Ny}$, chosen by comparing the filtered cubes with the original ones. For lower values of $v$, besides the removal of spatial noise, the filter excludes also emission from the nucleus of the galaxy.

\subsection{Spectral fitting}

In order to measure the emission-line fluxes and the stellar and gas kinematics from the MaNGA data cubes, we used the Gas AND Absorption Line Fitting (GANDALF) code (Sarzi et al. 2006; Oh et al. 2011). In brief, the GANDALF code fits the emission and absorption lines simultaneously, allowing the separation of the relative contribution of the stellar continuum and of nebular emission in the spectra of the galaxies. To subtract the underlying stellar contribution on the spectra of the galaxy and measure the stellar kinematics, GANDALF uses the Penalized Pixel-Fitting (PPXF) routine (Cappellari \& Emsellem 2004; Cappellari 2017). The continuum spectra of the galaxy are fitted using a library of template spectra under the assumption that the line-of-sight velocity distribution (LOSVD) of the stars is well reproduced by a GaussHermite series.

As template spectra, we used 30 selected evolutionary population synthesis models from Bruzual \& Charlot (2003), covering ages ranging from $5 \mathrm{Myr}$ to $12 \mathrm{Gyr}$ and three metallicities 
$\left(0.004 Z_{\odot}, 0.02 Z_{\odot}\right.$, and $\left.0.05 Z_{\odot}\right)$. During the fit of the spectra, we allowed the use of an order 3 multiplicative Legendre polynomial to correct the shape of the continuum and only the first two GaussHermite moments (velocity and velocity dispersion) were included to represent the LOSVD. We have tested the inclusion of higher order moments, but achieved the best results in the fitting process by considering only the first and second moments.

The emission-line profiles were fitted by Gaussian curves, by keeping tied the centroid velocity and width of the $[\mathrm{N}$ II $] \lambda \lambda 6548$, 6583 and [S II] $\lambda \lambda 6716,6731$ emission lines, fitting each doublet separately. In addition, the following line flux-ratio was kept fixed to their theoretical value: $[\mathrm{N} \mathrm{II}] \lambda 6583 /[\mathrm{N}$ II $] \lambda 6548=2.94$ (Osterbrock \& Ferland 2006). GANDALF gives output measurements for the centroid velocity and velocity dispersion $(\sigma)$ of the stars, and the flux, centroid velocity, and $\sigma$ of the emission lines for each spaxel, used to construct 2D maps.

\subsection{Measurements of the kinematic position angles}

In order to measure the global kinematic PA (i.e. the orientation of line of nodes $-\Psi_{0}$ ) from the stellar and gas velocity fields, we used the kinemetry method (Krajnović et al. 2005). This method extracts general kinematic properties of the galaxies by the symmetrization of the observed velocity fields, without the need of any assumption on the geometry of the stellar distribution. To obtain the global kinematic PA, the kinemetry method performs the symmetrization of the observed velocity fields. For each possible PA a symmetric velocity field $V^{\prime}(x, y)$ is created, with the PA oriented along the $x$ axis. The symmetric velocity field is obtained by changing the mean velocity of each bin for the weighted average of the corresponding velocity in the four quadrants of the velocity field. The global kinematic PA is the one that minimizes $\chi^{2}=\sum_{n=1}^{N}\left(V^{\prime}(x, y)-\right.$ $V(x, y) / \Delta \mathrm{V})^{2}$, where $V(x, y)$ is the value of observed velocity field at the position $(x, y)$.

We used the IDL routine fit_kinematic_pa.pro, ${ }^{1}$ which is an implementation of the kinemetry method and allows the measurement of the global kinematic PA and systemic velocity of the galaxy from the observed velocity fields. The routine is an implementation of the method presented in appendix C of Krajnović et al. (2006) and has been used to study the stellar kinematics of large samples of galaxies, for example the SAURON (Cappellari et al. 2007) and ATLAS $^{3 \mathrm{D}}$ (Krajnović et al. 2011) surveys.

\section{RESULTS}

We have performed measurements for the stellar and gas kinematics and emission-line fluxes for $\mathrm{H} \beta$, [OIII] $\lambda 5007, \mathrm{H} \alpha$, [N II] $\lambda \lambda 6549,83$, and $[\mathrm{S}$ II] $\lambda \lambda 6716,31$. With the aim of testing our measurements, we have compared the emission-line fluxes, centroid velocities, and velocity dispersions with measurements provided by the MaNGA Data Analysis Pipeline (DAP - Westfall et al., in preparation), as part of the MPL-7.

Fig. 1 shows an example of our measurements (top row) compared with those from the DAP (bottom row) for the AGN mangaid 1-339163. The first column shows a map of the continuum emission, the following columns exhibit maps of emission line fluxes for [O III $] 5007 \AA$, H $\alpha$, and [N II]6583 , respectively. The comparison

\footnotetext{
${ }^{1}$ This routine was developed by M. Cappellari and is available at http:
} //www-astro.physics.ox.ac.uk/ $\sim \mathrm{mxc} /$ software. between the top and bottom rows shows that our flux measurements are similar to those provided by the DAP.

In Fig. 2, we show the velocity fields for the same galaxy mangaid 1-339163. We present the stellar velocity field together with the gas velocity fields derived for the same emission lines presented in Fig. 1. For comparison, we show our results in the top row, while the results from DAP are shown in the bottom row. The comparison shows that the two velocity fields are similar, although the DAP maps are noisier.

The comparison of the velocity dispersion maps obtained by us and from the DAP is shown in Fig. 3, following the same pattern of organization as the previous figures. As for the centroid velocity and emission-line flux maps, the $\sigma$ maps from DAP are noisier than ours. The gas and stellar $\sigma$ values will be used to search for outflows in the central region of the galaxies of our sample.

As noticed in Figs 1-3, our measurements are in general consistent with those provided by DAP, but the spatial filtering of the data allows the exclusion of spurious data, as clearly seen in the maps for the $[\mathrm{N} \mathrm{II}] 6583 \AA$ and $\mathrm{H} \alpha$ velocity dispersion, for which the maps constructed using the DAP show a spurious feature at 4 arcsec east of the nucleus, which is not present in our measurements. On the other hand, the DAP has the advantage of providing measurements for all emission lines present in the galaxy spectra, while we fit only the strongest lines. However, a detailed comparison of our measurements and those provided by DAP is beyond the scope of this paper.

In order to verify if outflows of gas from the central AGN affect significantly the kinematics of AGN hosts, we can compare the kinematic PA $\left(\Psi_{0}\right)$ of the gas and stellar velocity fields. The motion of the stars is dictated by the gravitational potential of the galaxy, while for the gas, an additional component due to outflows is expected for the AGN. By comparing the difference between the $\Psi_{0}$ values derived from the gas and stellar velocity fields for AGN and control samples, one should expect larger differences for the AGN if strong outflows are present. We derived $\Psi_{0}$ for the stellar and gas velocity fields using [O III] $5007 \AA$, H $\alpha$, and [N II]6583 emission lines. In Fig. 4, we show two examples of the observed and symmetrized velocity fields for two AGN (mangaid 1-95092 and mangaid 1-351790). This figure illustrates two distinct results: (i) the $\Psi_{0}$ from distinct emission-line velocity fields is very similar to each other for both galaxies, (ii) for the galaxy mangaid 1-95092 the $\Psi_{0}$ derived from the stellar velocity field is very similar to that derived for the gas velocity field, and (iii) in the case of the AGN host mangaid 1-351790 the orientation of the kinematic major axis of the stellar and gas velocity fields shows a significant offset. In Fig. 5, we show a similar figure for two control galaxies: mangaid 12-129446 and mangaid 1-178838, showing similar results as those observed for the AGN: similar $\Psi_{0}$ for all emission lines and in one case a distinct $\Psi_{0}$ for the gas and stars. From Figs 4 and 5, we can conclude that for these galaxies both AGN and controls present a rotation pattern in the stellar as well as in the gas velocity fields. In Table A1, we present the kinematic PA derived for all galaxies of our sample and the properties of AGN and control galaxies are shown in Table A2 and Table A3 (Rembold et al. 2017), respectively.

\section{DISCUSSION}

In order to investigate if the AGN feedback in our sample is powerful enough to disturb the gas kinematics on galactic scales and change the orientation of the kinematic major axis of the galaxy, we calculated the frequency of occurrence of a given PA offset in the AGN and control samples. We computed the difference in the 
$1-339163$

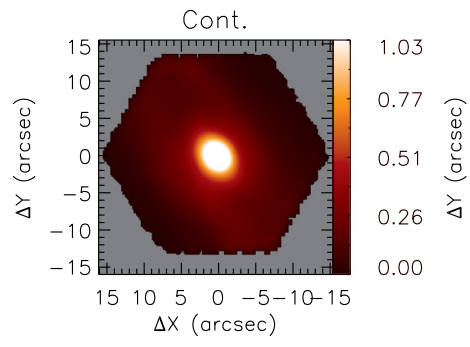

Cont.



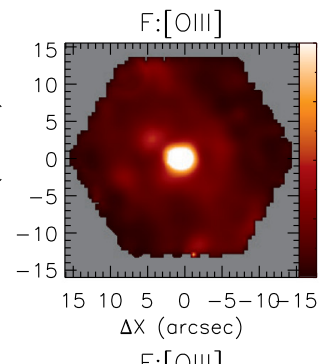

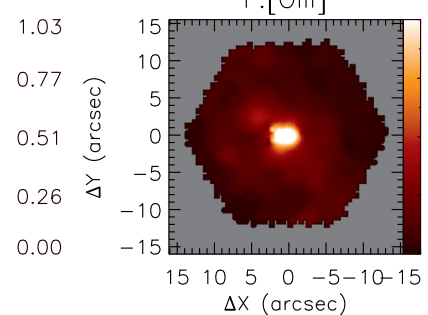

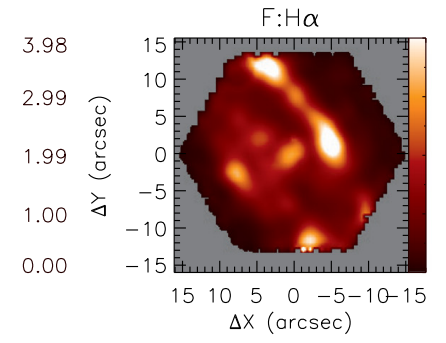

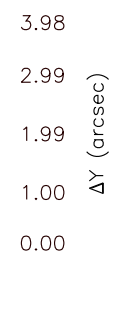

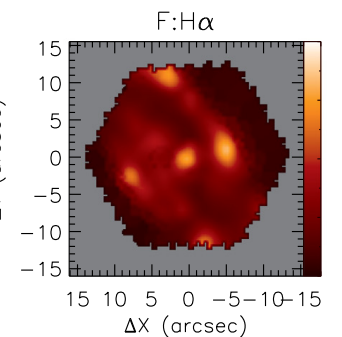
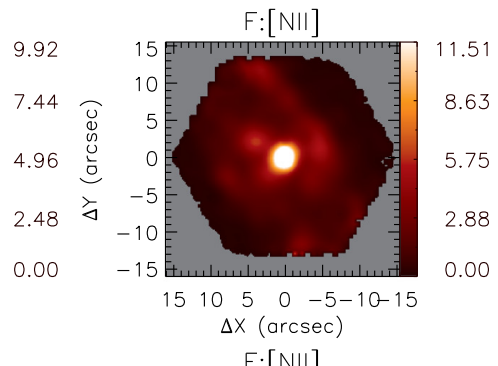

$\mathrm{F}:[\mathrm{NII}]$

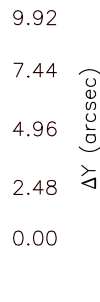

Figure 1. Emission lines fluxes for the galaxy with mangaid 1-339163. Our measurements are shown at the top row and the MaNGA-DAP measurements at the bottom row. In all panels, the North points up and East to the left and the $x$ and $y$ labels show the distance relative to the peak of the continuum emission. The first column shows a map of the continuum emission obtained by collapsing the whole spectral range, the following columns exhibit the spatial distribution of the emission line fluxes for [O III] $5007 \AA, \mathrm{H} \alpha$, and [N II] $6583 \AA$, respectively. The colour bars show the fluxes in unit of $10^{-17} \mathrm{erg} \mathrm{s}^{-1} \mathrm{~cm}^{-2} \mathrm{spx}^{-1}$.

\section{$1-339163$}
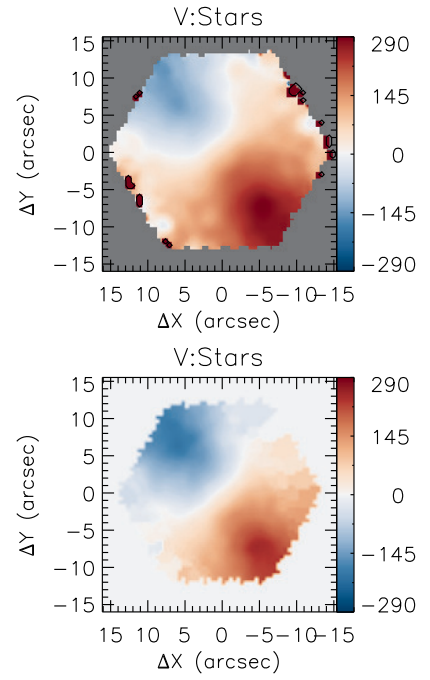
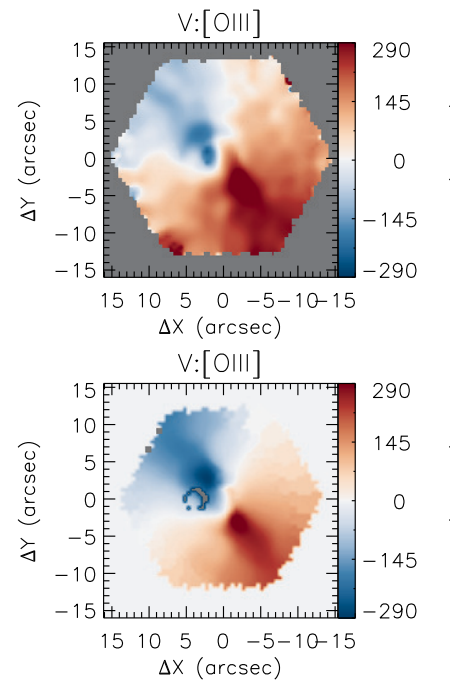


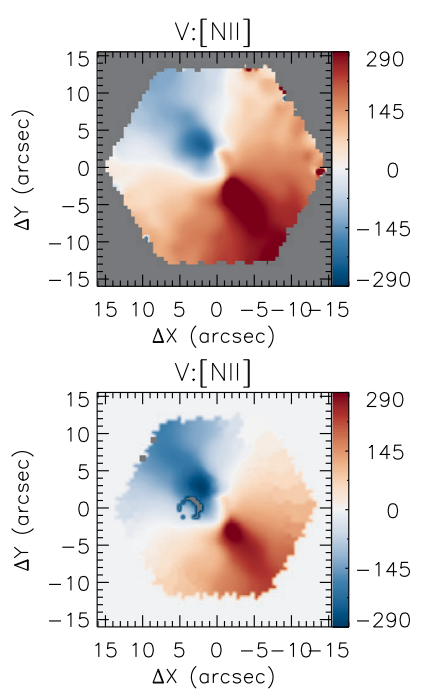

$\Delta \mathrm{X}(\operatorname{arcsec})$

Figure 2. Velocity fields for the galaxy mangaid 1-339163. Our measurements are shown at the top row and the DAP measurements at the bottom row. In all panels, the North points up and East to the left and the $x$ and $y$ labels show the distance relative to the peak of the continuum emission. The systemic velocity has been subtracted from each panel. The first column shows the stellar velocity field and the following columns exhibit the velocity fields for [O III], H $\alpha$, and $[\mathrm{N} \mathrm{II}]$, respectively. The velocity maps are in unit of $\mathrm{km} \mathrm{s}^{-1}$ relative to the systemic velocity of the galaxy.

$\Psi_{0 \star}$ of the stellar velocity field with respect to the $\Psi_{0 \text { gas }}$ derived for

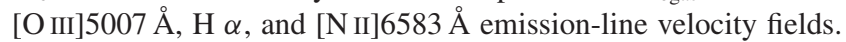
The resulting histograms of the PA offsets $\left(\triangle \mathrm{PA}=\left|\Psi_{0 \text { gas }}-\Psi_{0 \star}\right|\right)$ are presented in Fig. 6. The top panels show the results using the [O III] velocity fields, while the middle panel shows these results for $\mathrm{H} \alpha$ and the bottom panel for [N II]. AGN are represented by the blue colours and control galaxies are shown in red.

We find no clear difference in the distribution of $\triangle \mathrm{PA}$ for the AGN and control samples. Similar values of $\triangle \mathrm{PA}$ are observed for distinct emission lines. Although a few galaxies display large $\triangle \mathrm{PA}$ values, for most of them $\triangle \mathrm{PA}$ is smaller than $30^{\circ}$. For 79 per cent of AGN and 81 percent of control galaxies, the PA offsets are smaller than $30^{\circ}$ as measured using the [O III] $5007 \AA$ velocity field as representative of the gas velocity field. This result indicates that the AGN feedback is not strong enough to disturb - more than in a control sample - the gas kinematics on the galactic scales probed by MaNGA. Indeed, the sample of active galaxies used here is composed mainly by low-luminosity AGN (Rembold et al. 2017), for which outflows from the accretion disc are expected to be weak, and thus the gas velocity fields of these AGN hosts on galactic scales are expected to be driven by the gravitational potential of the galaxy. Besides that, Wylezalek et al. (2017) only find evidence for 

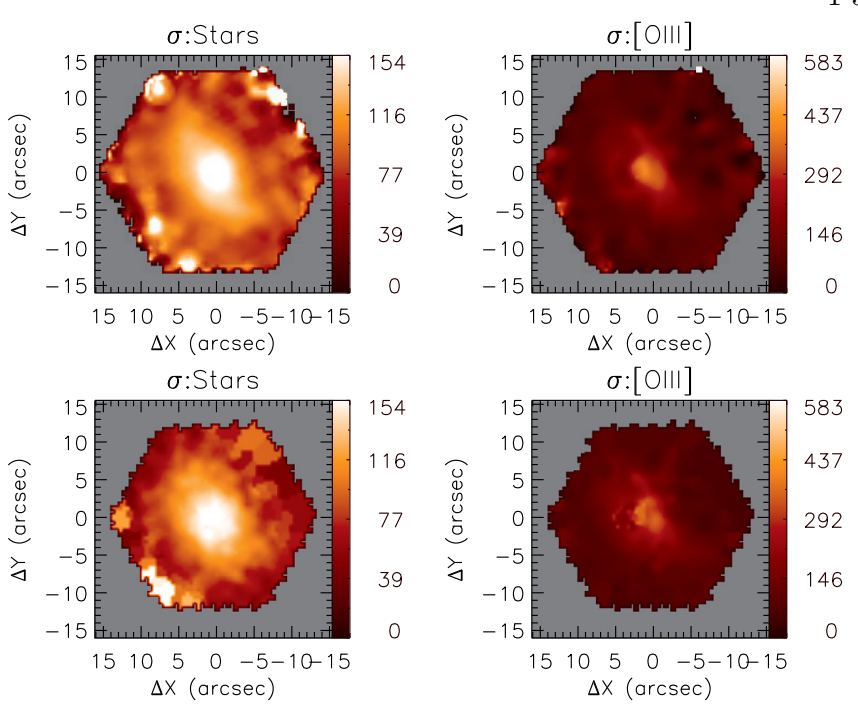

$1-339163$
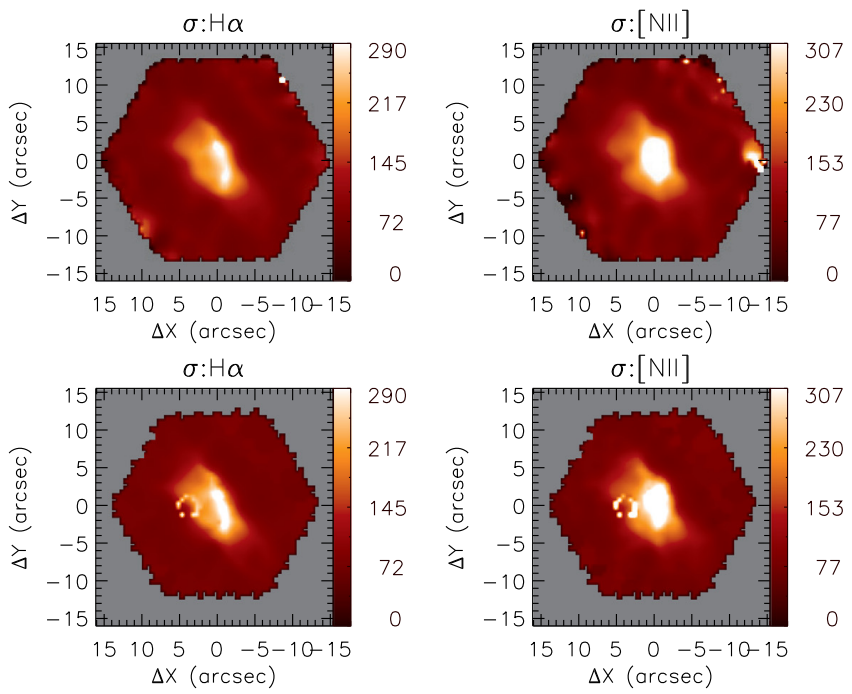

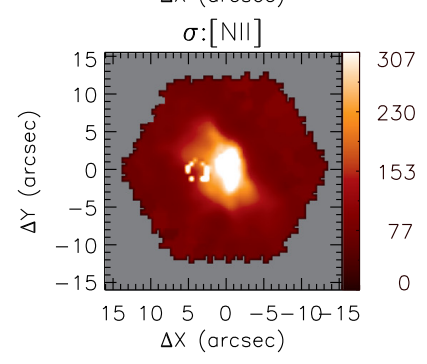

Figure 3. Velocity dispersion maps for the galaxy with mangaid 1-339163. Our measurements are shown at the top row and the DAP measurements at the bottom row. In all panels, the North points up and East to the left and the $x$ and $y$ labels show the distance relative to the peak of the continuum emission. The first column shows the stellar velocity dispersion distribution and the following columns exhibit the gas velocity dispersion distributions for [O III], H $\alpha$, and [N II], respectively. The colour bars show the velocity dispersion corrected by instrumental broadening in units of $\mathrm{km} \mathrm{s}^{-1}$.

an AGN-driven outflow in a MaNGA-selected AGN candidate when zoom into the centre with higher spatial resolution. The resolution of MaNGA is only 1 1".5-2".5, so a lot of small-scale outflows may be hidden. We do not find any clear difference in the $\triangle \mathrm{PA}$ of highand low-luminosity AGN.

Penny et al. (2018) analysed low-mass galaxies $\left(\mathrm{M}_{\star} \lesssim\right.$ $\left.5 \times 10^{9} \mathrm{M}_{\odot}\right)$ of the SDSS-IV MaNGA and found that five galaxies of their sample of 13 possible dwarf AGN host, exhibit ionized gas components in $\mathrm{H} \alpha$ that are kinematically offset from their stellar velocity field and these objects have AGN-like emission line ratios at their centres. This fact has been interpreted as a recent accretion episode or outflow. Furthermore, Penny et al. (2018) suggest that AGN feedback may play an important role in these low-mass galaxies. Their sample can be considered an analogous of the 'Red Geysers' galaxies reported by Cheung et al. (2016) using MaNGA data. These galaxies do not show recent star formation activity, most of them harbour very low-luminosity AGN, showing large-scale bi-polar outflows in ionized gas and interpreted as being originated by centrally driven winds due to a radiatively inefficient accretion flow on to the SMBH. These galaxies show stellar and gas kinematic major axes misaligned and account for 10 per cent of the population of galaxies with masses of the order of $2 \times 10^{10} \mathrm{M}_{\odot}$ that do not show recent star formation episodes. Although some galaxies of our sample show $\triangle \mathrm{PA}>30^{\circ}$, as seen in Fig. 6, the fraction of AGN and control galaxies with significant PA offset is similar (21 percent and 19 per cent for AGN and control sample, respectively), suggesting that these offsets are not associated with the presence AGN, and probably they are just statistical fluctuations. Thus, we show that standard AGN do not follow the same behaviour of 'Red Geyser' galaxies analysed by Cheung et al. (2016) and the low-mass galaxies presented in Penny et al. (2018), as we do not detect significant PA offsets.

The fact that there are no significant PA offsets in our sample does not necessarily mean that the AGN do not show outflows, although it implies they do not play an important role in the galaxy scale gas kinematics. However, AGN-driven outflows could be seen on smaller scales.

In order to search for signatures of outflows closer to the nuclei of the galaxies, we have compared the stellar and gas velocity dispersion values within the inner 2".5 diameter of the galaxies of our sample, as this aperture corresponds to the angular resolution of the MaNGA data cubes. In Table A1, we show these velocity dispersion values. On average, the 2 .'5 aperture corresponds to a physical scale of $\sim 2 \mathrm{kpc}$ at the typical redshift of the sample galaxies. In order to quantify the differences between the stellar and gas velocity dispersions measured in the central regions, we calculated the parameter $\sigma_{\text {frac }}$, defined as

$\sigma_{\mathrm{frac}}=\frac{\sigma_{\mathrm{gas}}-\sigma_{\star}}{\sigma_{\star}}$,

which measures the fractional difference between the gas and stellar velocity dispersion, and thus higher values of $\sigma_{\text {frac }}$ are indicative of a disturbed kinematics (not only due to the gravitational potential of the galaxy) and most probably due to outflows.

We see a trend of AGN having generally higher $\sigma_{\text {frac }}$ values than inactive galaxies as can be seen in the distributions shown in Fig. 7. The median values of $\sigma_{\text {frac }}$ for AGN and control sample are $<\sigma_{\text {frac }}>_{\text {AGN }}=0.04$ and $\left\langle\sigma_{\text {frac }}>_{\text {CTR }}=-0.23\right.$, respectively. Besides that, we note that 90 percent of AGN have $\sigma_{\text {frac }}$ larger than -0.22 and 75 per cent of them have values larger than -0.13 . For the control sample, 90 percent of the galaxies show $\sigma_{\text {frac }}<$ 0.12 and for 75 percent of the sample $\sigma_{\text {frac }}<-0.04$. The result of the Anderson-Darling statistical test returns a p-value of $10^{-5}$, which confirms that the AGN and inactive galaxies follow distinct distributions in $\sigma_{\text {frac }}$. We thus conclude that the parameter $\sigma_{\text {frac }}$ can be used as an indicator of AGN activity.

We derived the luminosity of the [O III] $25007 \AA$ emission line $\left(L_{[\mathrm{OIII}}\right)$ of each galaxy (Table A1) using the flux measurements obtained with the GANDALF code within the same aperture used to measure the $\sigma_{\text {frac }}$, and then investigated a possible correlation 

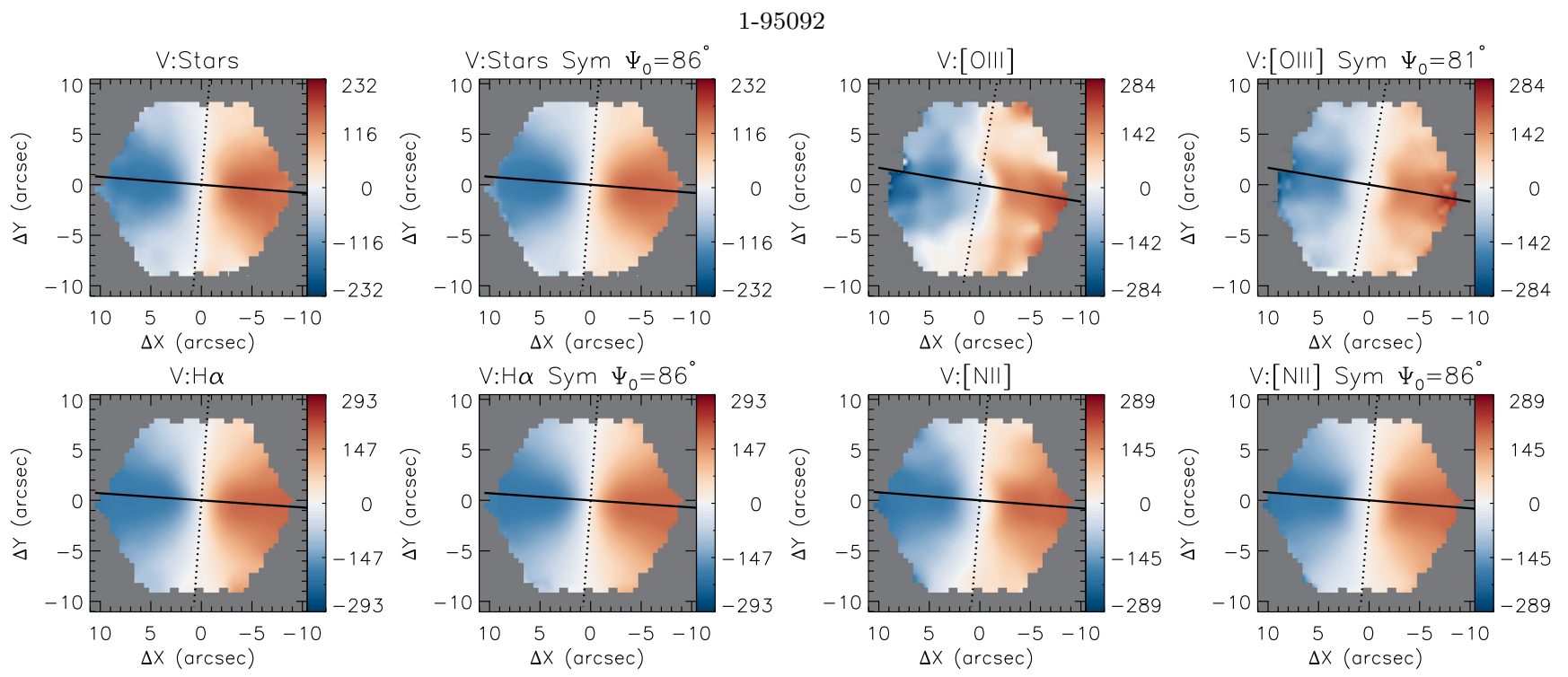

$1-351790$


Figure 4. In the first two rows, we show the derived velocity fields for the AGN mangaid 1-95092. The first row shows, from left to right, the stellar velocity field (V:Stars), the symmetrized stellar velocity field (V:Stars Sym), the gas velocity field for [O III] (V:[O III]), and the corresponding symmetrized velocity field (V:[O III] Sym). The second row shows, from left to right, the $\mathrm{H} \alpha$ velocity field (V:H $\alpha)$, its symmetrized velocity field (V:H $\alpha$ Sym), the velocity and symmetrized velocity fields for [N II] (V:[N III]) and (V:[N II] Sym), respectively. In the bottom two rows, we show the same velocity maps but for the AGN mangaid 1-351790. In all velocity maps, the solid black line shows the PA of kinematic major axis, the value of the $\Psi_{0}$ is shown in the top right-hand corner of the symmetrized velocity maps. The continuous (dotted) line shows the orientation of the kinematic major (minor) axis of the galaxy. The colour bars show the velocities in units of $\mathrm{km} \mathrm{s}^{-1}$.

between $\sigma_{\text {frac }}$ and $L_{[\mathrm{O} \text { III] }}$. Fig. 8 shows the plot of $L_{[\mathrm{O} \mathrm{III}]}$ versus $\sigma_{\text {frac }}$ for the AGN and control samples. There is a clear positive correlation between $\sigma_{\text {frac }}$ and $L_{[\mathrm{OIII}]}$, with a Spearman correlation coefficient of 0.53 and a p-value of $10^{-14}$. However, it should be noticed that the observed correlation could be artificially produced, as the AGN and inactive galaxies clearly show distinct distributions in $\sigma_{\text {frac }}$ (Fig. 7). The Spermann test returns a p-value of 0.06 for the AGN sample and $10^{-5}$ for the control sample, meaning that no strong correlation is found between the $L_{[\mathrm{O} \text { III }]}$ and $\sigma_{\text {frac }}$ for the AGN sample alone, while these parameters are correlated for the control sample. The absence of correlation for the AGN sample may be due to the fact that our sample covers only a small range of luminosities, as most objects are low-luminosity AGN (Rembold et al. 2017). Fig.
7 shows a trend of AGN having higher $\sigma_{\text {frac }}$ values than inactive galaxies. The same trend can also be observed in Fig. 8. This result can be interpreted as the higher values seen for AGN compared to control galaxies being due to winds originated in the AGN. Thus, although the AGN of the sample do not show powerful outflows that can affect the gas kinematics on galactic scales, they do show small-scale outflows (within the inner 1-2 kpc).

Our results can be compared with those obtained from single aperture spectra. For example, Woo et al. (2017) find that there is a trend of the $[\mathrm{O}$ III] $] 5007 \AA$ velocity dispersion to increase with the increase in the AGN luminosity in a sample of $\sim 110000$ AGN and star-forming $(\mathrm{SF})$ galaxies at $z<0.3$. This trend is also present in composite objects and is not clear for SF galaxies. They interpreted 



$2-129446$
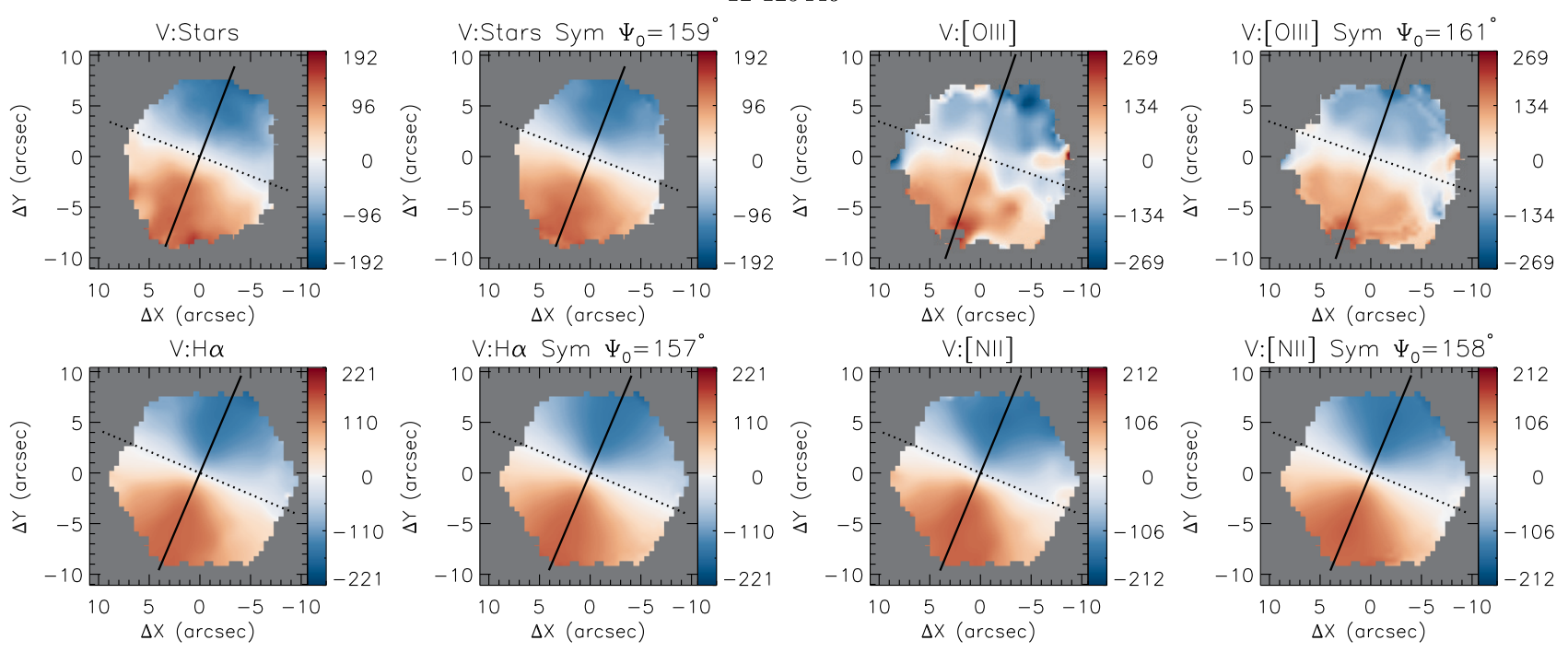

$1-178838$


Figure 5. Same as Fig. 4 for the control galaxies mangaid 12-129446 (first and second row) and mangaid 1-178838 (third and fourth row). The velocity fields are in the unit of $\mathrm{km} \mathrm{s}^{-1}$.

this result due to strong gas outflows in high-luminosity AGN, indicating that AGN energetics are driving these outflows. They find also lower average [O III] velocity dispersion values for SF galaxies. Our result is in good agreement with theirs. In addition, optical observations (Wylezalek et al. 2016), radio observations (Zakamska \& Greene 2014), and molecular gas (Veilleux et al. 2013) as well as theoretical models (Zubovas \& King 2012) have suggested that the AGN need to have enough luminosity for the gas to be pushed out of the galactic potential. This is in agreement with our results, where we see a positive correlation between $\sigma_{\text {frac }}$ and luminosity.

\section{CONCLUSIONS}

We have mapped the gas and stellar kinematics of a sample of 62 AGN and 109 control galaxies (inactive galaxies) in order to investigate the effect of the AGN in the large- and small-scale gas kinematics of the AGN host galaxies. We detect evidence of nuclear gas outflows in the 62 AGN, but conclude they are not powerful enough to play an important role in the gas kinematics on galactic scales. The main conclusions of our work are as follows:

(i) There is no significant difference in the $\triangle \mathrm{PA}$ between active and inactive galaxies, indicating that the galaxy scale gas kinematics are dominated by orbital motion in the gravitational potential of the galaxies, instead of outflows driven by the central AGN.

(ii) We found that the difference between the orientation of the kinematic major axes of the gas and stars $(\triangle \mathrm{PA})$ is larger than $30^{\circ}$ for 13 (21 percent) AGN and 21 control galaxies (19 percent) using the [O III] $5007 \AA$ kinematics.

(iii) The AGN show larger fractional differences in the velocity dispersions of the gas and stars $\sigma_{\text {frac }}=\frac{\sigma_{\mathrm{O} \amalg}-\sigma_{\star}}{\sigma_{\star}}$ than inactive galaxies within the inner 2.5 diameter, which corresponds to $1-2 \mathrm{kpc}$ at 

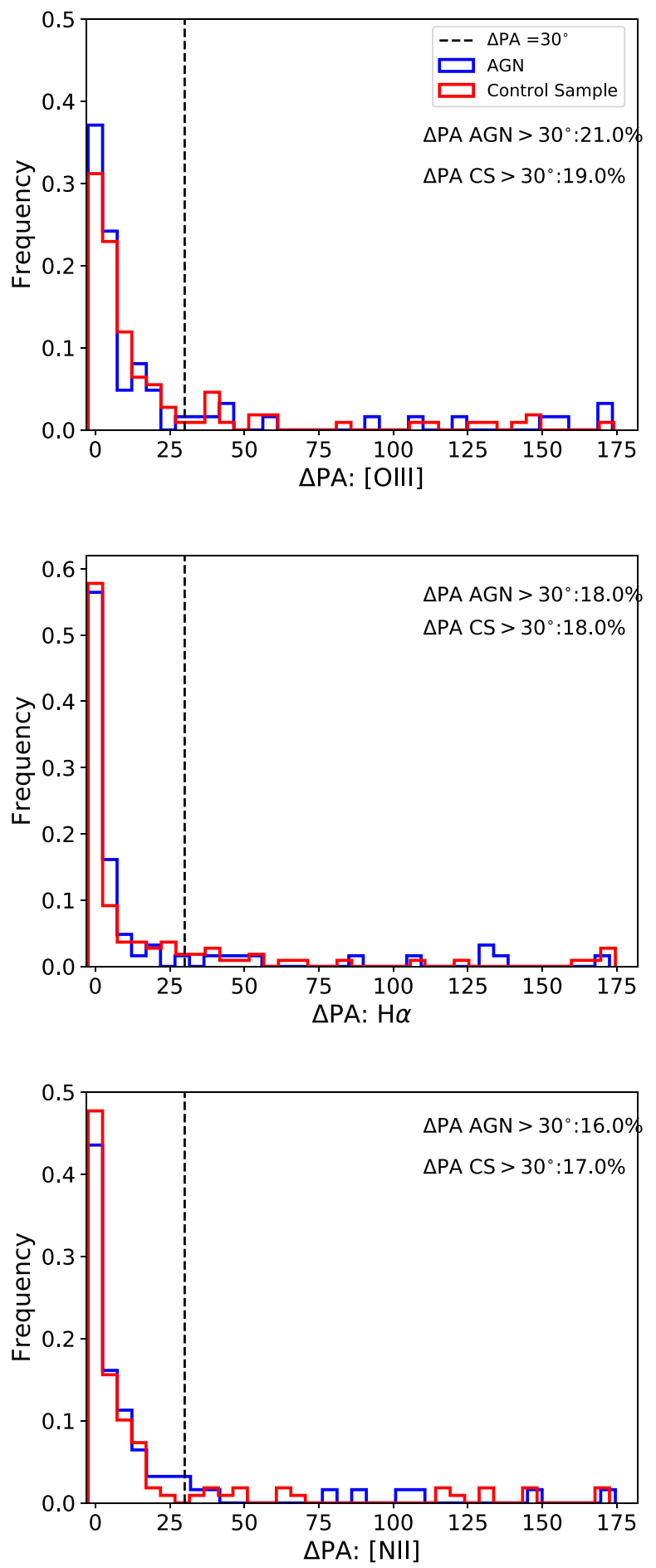

Figure 6. Histograms comparing $\triangle \mathrm{PA}$ distributions of AGN and control galaxies for [O III] $\lambda 5007$ (top panel), $\mathrm{H} \alpha$ (middle panel), and [N II] $] 6583$ (bottom line). AGN are shown in blue and controls in red. The vertical dashed lines show $\triangle \mathrm{PA}=30^{\circ}$.

the galaxies. The mean values are $\sigma_{\text {frac }}=0.04$ for the AGN and $\sigma_{\text {frac }}=-0.23$ for the control sample. This difference is interpreted as being due to outflows from the active nuclei. This indicates that although the AGN of our sample do not affect the gas kinematics on large scale, it does affect it at least within the inner kpc.

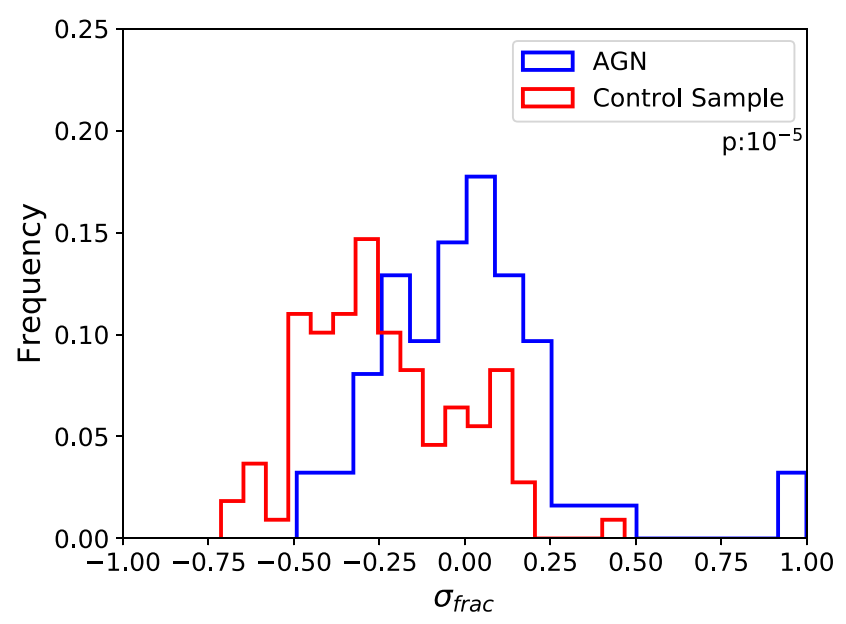

Figure 7. Histograms comparing $\sigma_{\text {frac }}$ distributions of AGN and control galaxies for $[\mathrm{O} \mathrm{III}] \lambda 5007$. AGN are shown in blue and controls in red. The result of Anderson-Darling statistical test returns a p-value of $10^{-5}$, confirming that AGN and inactive galaxies follow distinct distributions in $\sigma_{\text {frac }}$.

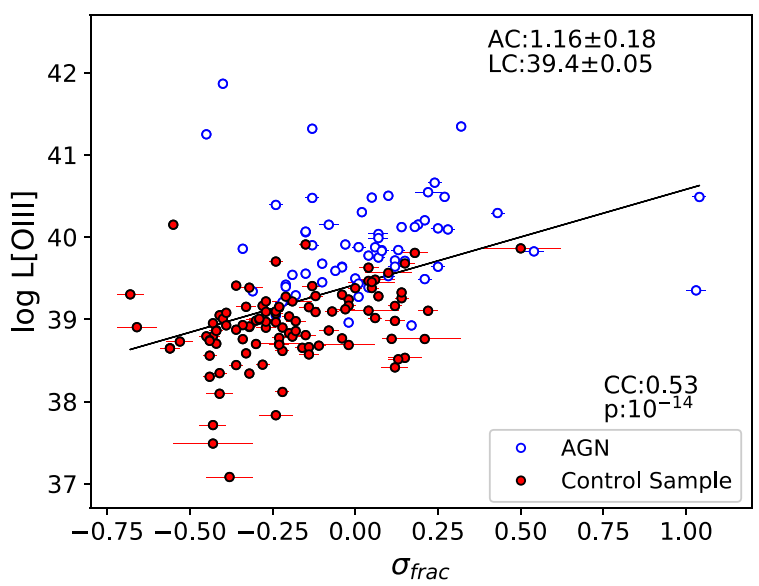

Figure 8. Plot of logarithm of $[\mathrm{O}$ III $] 5007 \AA$ luminosity versus $\sigma_{\text {frac }}$ for AGN (blue open circles) and inactive galaxies (red closed circles). The Spearman test confirms that these properties are correlated resulting in a correlation coefficient of 0.53 and p-value of $10^{-14}$. The black line is the result of linear fit of data, with linear coefficient of $L C=39.4 \pm 0.05$ and angular coefficient of $A C=1.16 \pm 0.18$.

(iv) A correlation between the [O III $] 5007 \AA$ luminosity and $\sigma_{\text {frac }}$ is observed when putting together the AGN and control samples.

\section{ACKNOWLEDGEMENTS}

Funding for the Sloan Digital Sky Survey IV has been provided by the Alfred P. Sloan Foundation and the Participating Institutions. SDSS-IV acknowledges support and resources from the Center for High-Performance Computing at the University of Utah. The SDSS web site is www.sdss.org. SDSS-IV is managed by the Astrophysical Research Consortium for the Participating Institutions of the SDSS Collaboration including the Brazilian Participation Group, the Carnegie Institution for Science, Carnegie Mellon University, the Chilean Participation Group, Harvard-Smithsonian Center for Astrophysics, Instituto de Astrofísica de Canarias, The Johns 
Hopkins University, Kavli Institute for the Physics and Mathematics of the Universe (IPMU) / University of Tokyo, Lawrence Berkeley National Laboratory, Leibniz Institut für Astro physik Potsdam (AIP), Max-Planck-Institut für Astrophysik (MPA Garching), MaxPlanck-Institut für Extraterrestrische Physik (MPE), Max-PlanckInstitut für Astronomie (MPIA Heidelberg), National Astronomical Observatory of China, New Mexico State University, New York University, The Ohio State University, Pennsylvania State University, Shanghai Astronomical Observatory, United Kingdom Participation Group, Universidad Nacional Autónoma de México, University of Arizona, University of Colorado Boulder, University of Portsmouth, University of Utah, University of Washington, University of Wisconsin, Vanderbilt University, and Yale University. We thank the support of the Instituto Nacional de Ciência e Tecnologia (INCT) e-Universe (CNPq grant 465376/2014-2).

This study was financed in part by the Coordenação de Aperfeiçoamento de Pessoal de Nível Superior - Brasil (CAPES) - Finance Code 001, Conselho Nacional de Desenvolvimento Científico e Tecnológico $(\mathrm{CNPq})$, and Fundação de Amparo à pesquisa do Estado do RS (FAPERGS).

\section{REFERENCES}

Abolfathi B. et al., 2018, ApJS, 235, 42

Antonucci R., 1993, ARA\&A, 31, 473

Barbosa F. K. B., Storchi-Bergmann T., McGregor P., Vale T. B., Riffel R. A., 2014, MNRAS, 445, 2353

Bower R. G., Benson A. J., Malbon R., Helly J. C., Frenk C. S., Baugh C. M., Cole S., Lacey C. G., 2006, MNRAS, 370, 645

Bruzual G., Charlot S., 2003, MNRAS, 344, 1000

Bundy K. et al., 2015, ApJ, 798, 7

Cappellari M., 2017, MNRAS, 466, 798

Cappellari M., Emsellem E., 2004, PASP, 116, 138

Cappellari M. et al., 2007, MNRAS, 379, 418

Cheung E. et al., 2016, Nature, 533, 504

Conroy C., Gunn J. E., White M., 2009, ApJ, 699, 486

Di Matteo T., Springel V., Hernquist L., 2005, Nature, 433, 604

Drory N. et al., 2015, AJ, 149, 77

Ferrarese L., Merritt D., 2000, ApJ, 539, L9

Fischer T. C., Crenshaw D. M., Kraemer S. B., Schmitt H. R., 2013, ApJS, 209,1

Gebhardt K. et al., 2000, ApJ, 539, L13

Gonzalez R. C., Woods R. E., 2002, Digital Image Processing, 2nd edn. Prentice-Hall, Upper Saddle River, NJ
Hopkins P. F., Hernquist L., Cox T. J., Di Matteo T., Martini P., Robertson B., Springel V., 2005, ApJ, 630, 705

Krajnović D., Cappellari M., Emsellem E., McDermid R. M., de Zeeuw P. T., 2005, MNRAS, 357, 1113

Krajnović D., Cappellari M., de Zeeuw P. T., Copin Y., 2006, MNRAS, 366, 787

Krajnović D., Cappellari M., de Zeeuw P. T., Copin Y., 2011, MNRAS, 414, 2993

Law D. R. et al., 2015, AJ, 150, 19

Law D. R. et al., 2015, AJ, 152, 83

Lena D. et al., 2015, ApJ, 806, 84

Mallmann N. D., et al., 2018, MNRAS, 478, 5491 (Paper II)

Oh K., Sarzi M., Schawinski K., Yi S. K., 2011, ApJS, 195, 13

Osterbrock D. E., Ferland G. J., 2006, Astrophysics of Gaseous Nebulae and Active Galactic Nuclei, 2nd edn. University Science Books, Sausalito, California

Penny S. J. et al., 2018, MNRAS, 476, 979

Rembold S. B. et al., 2017, MNRAS, 472, 4382 (Paper I)

Riffel R. A., Storchi-Bergmann T., Riffel R., 2014, ApJ, 780, L24

Sarzi M. et al., 2006, MNRAS, 366, 1151

Scannapieco E., Silk J., Bouwens R., 2005, ApJ, 635, L13

Schmitt H. R., Donley J. L., Antonucci R. R. J., Hutchings J. B., Kinney A. L., 2003, ApJS, 148, 327

Schnorr-Müller A., Storchi-Bergmann T., Nagar N. M., Robinson A., Lena D., Riffel R. A., Couto G. S., 2014, MNRAS, 437, 1708

Springel V., Di Matteo T., Hernquist L., 2005, ApJ, 620, L79

Tremaine S. et al., 2002, ApJ, 574, 740

Urry C. M., Padovani P., 1995, PASP, 107, 803

Veilleux S. et al., 2013, ApJ, 776, 27

Wake D. A. et al., 2017, AJ, 154, 86

Woo J.-H., Son D., Bae H.-J., 2017, ApJ, 839, 120

Wylezalek D., Zakamska N. L., 2016, MNRAS, 461, 3724

Wylezalek D. et al., 2017, MNRAS, 467, 2612

Wylezalek D., Zakamska N. L., Greene J. E., Riffel R. A., Drory N., Andrews

B. H., Merloni A., Thomas D., 2018, MNRAS, 474, 1499

Yan R. et al., 2016a, AJ, 151, 8

Yan R. et al., 2016b, AJ, 152, 197

Zakamska N. L., Greene J. E., 2014, MNRAS, 442, 784

Zakamska N. L. et al., 2016, MNRAS, 459, 3144

Zubovas K., King A., 2012, ApJ, 745, L34

\section{APPENDIX A: KINEMATIC MEASUREMENTS AND PARAMETERS OF THE AGN AND CONTROL SAMPLE}


Table A1. Kinematic measurements of the AGN and control samples. Col. 1: mangaid; Col. 2: Logarithm of luminosity of [O III] $] 5007 \AA$ in erg s ${ }^{-1}$ within the inner 2.' 5 diameter; velocity dispersion in $\mathrm{km} \mathrm{s}^{-1}$ obtained for stars, [O III] $5007 \AA$, $\mathrm{H} \alpha$, [N II] $6583 \AA$ (cols. 3-6), measured within the inner 2'.5 diameter, and kinematic position angles $\left(\Psi_{0}\right)$ for the stars, [O III] $5007 \AA, \mathrm{H} \alpha,[\mathrm{N}$ II] $6583 \AA$ (cols. 7-10). In each block we have the parameters obtained for AGN (first line) and their controls (second and third lines).

\begin{tabular}{|c|c|c|c|c|c|c|c|c|c|}
\hline mangaid & $\log _{10} L_{[} \mathrm{O}$ III] & $\sigma_{\star}$ & $\sigma_{\text {[O III] }]}$ & $\sigma_{H \alpha}$ & $\sigma_{[\mathrm{N}}$ II $]$ & $\Psi_{0 \star}$ & $\Psi_{0}[\mathrm{O}$ III $]$ & $\Psi_{0} \mathrm{H} \alpha$ & $\Psi_{0}[\mathrm{~N} \mathrm{III}]$ \\
\hline $1-71481$ & 39.38 & $284.0 \pm 2.0$ & $194.0 \pm 12.0$ & $275.0 \pm 12.0$ & $219.0 \pm 11.0$ & $174.0 \pm 0.5$ & $146.5 \pm 0.5$ & $9.5 \pm 0.5$ & $163.5 \pm 0.5$ \\
\hline $1-269632$ & 41.35 & $153.0 \pm 1.0$ & $202.0 \pm 2.0$ & $137.0 \pm 2.0$ & $155.0 \pm 1.0$ & $16.0 \pm 2.2$ & $173.5 \pm 0.5$ & $11.5 \pm 2.0$ & $14.0 \pm 0.8$ \\
\hline $1-210700$ & 39.7 & $199.0 \pm 2.0$ & $150.0 \pm 4.0$ & $112.0 \pm 1.0$ & $131.0 \pm 1.0$ & $130.0 \pm 0.5$ & $137.5 \pm 0.5$ & $134.5 \pm 0.5$ & $119.5 \pm 0.5$ \\
\hline $1-258599$ & 41.87 & $349.0 \pm 3.0$ & $208.0 \pm 1.0$ & $170.0 \pm 1.0$ & $166.0 \pm 1.0$ & $81.5 \pm 0.5$ & $98.5 \pm 0.5$ & $122.5 \pm 0.5$ & $108.5 \pm 0.5$ \\
\hline $1-93876$ & 39.11 & $223.0 \pm 1.0$ & $230.0 \pm 20.0$ & $275.0 \pm 18.0$ & $98.0 \pm 18.0$ & $146.0 \pm 0.5$ & $148.5 \pm 0.5$ & $149.5 \pm 0.5$ & $136.0 \pm 0.5$ \\
\hline $1-166691$ & 38.76 & $231.0 \pm 1.0$ & $281.0 \pm 25.0$ & $175.0 \pm 15.0$ & $221.0 \pm 39.0$ & $92.0 \pm 0.5$ & $146.5 \pm 0.5$ & $34.5 \pm 0.5$ & $95.0 \pm 0.5$ \\
\hline $1-72322$ & 41.25 & $287.0 \pm 3.0$ & $156.0 \pm 2.0$ & $170.0 \pm 2.0$ & $182.0 \pm 3.0$ & $107.0 \pm 0.8$ & $114.5 \pm 0.5$ & $116.0 \pm 0.5$ & $118.5 \pm 0.5$ \\
\hline $1-121717$ & 39.86 & $223.0 \pm 2.0$ & $334.0 \pm 26.0$ & $167.0 \pm 3.0$ & $173.0 \pm 4.0$ & $134.0 \pm 0.5$ & $111.0 \pm 0.5$ & $134.0 \pm 0.5$ & $134.0 \pm 0.5$ \\
\hline $1-209980$ & 40.66 & $179.0 \pm 3.0$ & $218.0 \pm 1.0$ & $233.0 \pm 1.0$ & $245.0 \pm 1.0$ & $50.0 \pm 0.8$ & $52.0 \pm 0.5$ & $55.0 \pm 0.5$ & $51.5 \pm 0.5$ \\
\hline $1-295095$ & 38.76 & $101.0 \pm 1.0$ & $112.0 \pm 3.0$ & $80.0 \pm 1.0$ & $88.0 \pm 2.0$ & $1.0 \pm 2.2$ & $3.0 \pm 0.8$ & $178.0 \pm 1.5$ & $1.0 \pm 1.2$ \\
\hline $1-92626$ & 39.41 & $181.0 \pm 2.0$ & $116.0 \pm 2.0$ & $138.0 \pm 2.0$ & $183.0 \pm 10.0$ & $10.5 \pm 0.8$ & $13.5 \pm 0.5$ & $13.0 \pm 0.5$ & $13.0 \pm 0.5$ \\
\hline $1-44379$ & 40.49 & $133.0 \pm 2.0$ & $168.0 \pm 2.0$ & $126.0 \pm 3.0$ & $159.0 \pm 3.0$ & $15.0 \pm 0.5$ & $14.0 \pm 0.5$ & $16.5 \pm 0.5$ & $16.5 \pm 0.8$ \\
\hline $1-211082$ & 38.86 & $153.0 \pm 1.0$ & $89.0 \pm 3.0$ & $118.0 \pm 2.0$ & $158.0 \pm 1.0$ & $157.5 \pm 0.5$ & $163.5 \pm 0.5$ & $159.0 \pm 0.5$ & $159.5 \pm 0.5$ \\
\hline $1-135371$ & 38.9 & $144.0 \pm 2.0$ & $105.0 \pm 4.0$ & $116.0 \pm 2.0$ & $147.0 \pm 4.0$ & $67.5 \pm 0.8$ & $72.5 \pm 0.5$ & $66.5 \pm 0.8$ & $66.5 \pm 0.5$ \\
\hline $1-149211$ & 40.5 & $107.0 \pm 1.0$ & $116.0 \pm 0.0$ & $121.0 \pm 1.0$ & $134.0 \pm 2.0$ & $41.0 \pm 0.5$ & $166.0 \pm 0.5$ & $174.5 \pm 0.5$ & $25.5 \pm 0.5$ \\
\hline $1-377321$ & 40.15 & $182.0 \pm 6.0$ & $77.0 \pm 0.0$ & $77.0 \pm 0.0$ & $80.0 \pm 0.0$ & $17.5 \pm 0.5$ & $26.5 \pm 0.5$ & $44.0 \pm 0.5$ & $85.5 \pm 0.5$ \\
\hline $1-491233$ & 39.09 & $106.0 \pm 1.0$ & $94.0 \pm 1.0$ & $83.0 \pm 1.0$ & $87.0 \pm 1.0$ & $120.5 \pm 1.5$ & $105.0 \pm 1.8$ & $118.5 \pm 1.2$ & $118.5 \pm 1.5$ \\
\hline $1-279147$ & 40.29 & $116.0 \pm 1.0$ & $163.0 \pm 1.0$ & $101.0 \pm 1.0$ & $122.0 \pm 1.0$ & $31.5 \pm 4.2$ & $51.5 \pm 0.5$ & $54.0 \pm 0.5$ & $1.0 \pm 0.5$ \\
\hline $1-283246$ & 38.68 & $142.0 \pm 0.0$ & $126.0 \pm 3.0$ & $157.0 \pm 8.0$ & $140.0 \pm 3.0$ & $37.0 \pm 2.8$ & $59.0 \pm 1.0$ & $29.5 \pm 0.5$ & $31.0 \pm 0.5$ \\
\hline $1-351538$ & 39.26 & $109.0 \pm 1.0$ & $125.0 \pm 3.0$ & $69.0 \pm 0.0$ & $76.0 \pm 1.0$ & $160.5 \pm 16.2$ & $141.0 \pm 0.5$ & $169.0 \pm 1.2$ & $176.5 \pm 1.2$ \\
\hline $1-460812$ & 40.48 & $210.0 \pm 3.0$ & $179.0 \pm 2.0$ & $212.0 \pm 1.0$ & $218.0 \pm 3.0$ & $78.0 \pm 0.8$ & $78.5 \pm 0.5$ & $69.5 \pm 0.5$ & $68.5 \pm 0.5$ \\
\hline $1-270160$ & 39.63 & $319.0 \pm 3.0$ & $331.0 \pm 9.0$ & $362.0 \pm 17.0$ & $311.0 \pm 10.0$ & $111.0 \pm 0.5$ & $112.5 \pm 0.5$ & $104.0 \pm 0.5$ & $116.5 \pm 1.0$ \\
\hline $1-258455$ & 39.15 & $214.0 \pm 1.0$ & $184.0 \pm 7.0$ & $184.0 \pm 3.0$ & $194.0 \pm 7.0$ & $131.0 \pm 1.5$ & $130.0 \pm 0.5$ & $128.5 \pm 0.5$ & $127.5 \pm 0.5$ \\
\hline $1-92866$ & 40.3 & $241.0 \pm 1.0$ & $244.0 \pm 2.0$ & $217.0 \pm 1.0$ & $258.0 \pm 2.0$ & $18.5 \pm 1.5$ & $126.5 \pm 0.5$ & $151.0 \pm 0.5$ & $123.5 \pm 0.5$ \\
\hline $1-94514$ & 38.69 & $237.0 \pm 1.0$ & $229.0 \pm 18.0$ & $373.0 \pm 71.0$ & $386.0 \pm 23.0$ & $147.5 \pm 1.0$ & $147.0 \pm 0.5$ & $143.0 \pm 0.5$ & $135.0 \pm 0.5$ \\
\hline $1-210614$ & 38.91 & $223.0 \pm 1.0$ & $152.0 \pm 3.0$ & $178.0 \pm 2.0$ & $157.0 \pm 3.0$ & $131.0 \pm 2.2$ & $90.0 \pm 0.5$ & $95.0 \pm 0.5$ & $82.0 \pm 0.5$ \\
\hline $1-94784$ & 40.48 & $135.0 \pm 1.0$ & $142.0 \pm 2.0$ & $156.0 \pm 1.0$ & $153.0 \pm 2.0$ & $65.5 \pm 1.0$ & $71.0 \pm 0.5$ & $62.5 \pm 0.8$ & $67.5 \pm 1.5$ \\
\hline $1-211063$ & 38.75 & $136.0 \pm 1.0$ & $76.0 \pm 2.0$ & $141.0 \pm 2.0$ & $111.0 \pm 2.0$ & $170.0 \pm 1.8$ & $164.0 \pm 0.5$ & $166.5 \pm 1.8$ & $165.0 \pm 1.2$ \\
\hline $1-135502$ & 39.16 & $164.0 \pm 1.0$ & $117.0 \pm 1.0$ & $177.0 \pm 2.0$ & $163.0 \pm 1.0$ & $98.5 \pm 0.5$ & $98.5 \pm 0.5$ & $101.5 \pm 0.5$ & $102.0 \pm 0.5$ \\
\hline $1-44303$ & 40.15 & $114.0 \pm 1.0$ & $135.0 \pm 1.0$ & $120.0 \pm 2.0$ & $134.0 \pm 2.0$ & $56.0 \pm 7.2$ & $39.0 \pm 7.8$ & $67.5 \pm 2.0$ & $62.0 \pm 3.0$ \\
\hline $1-339028$ & 39.22 & $183.0 \pm 1.0$ & $133.0 \pm 2.0$ & $170.0 \pm 3.0$ & $145.0 \pm 1.0$ & $179.5 \pm 2.7$ & $173.5 \pm 1.0$ & $5.5 \pm 0.8$ & $9.0 \pm 1.8$ \\
\hline $1-379087$ & 39.27 & $131.0 \pm 1.0$ & $104.0 \pm 2.0$ & $123.0 \pm 1.0$ & $125.0 \pm 1.0$ & $39.5 \pm 2.2$ & $31.5 \pm 0.5$ & $42.5 \pm 0.5$ & $44.0 \pm 1.0$ \\
\hline
\end{tabular}


Table A1 - continued

\begin{tabular}{|c|c|c|c|c|c|c|c|c|c|}
\hline mangaid & $\log _{10} L_{[} \mathrm{O}$ III] & $\sigma_{\star}$ & $\left.\sigma_{[\mathrm{O}} \mathrm{III}\right]$ & $\sigma_{H \alpha}$ & $\left.\sigma_{[\mathrm{N}} \mathrm{II}\right]$ & $\Psi_{0 \star}$ & $\Psi_{0}[\mathrm{O} I I I]$ & $\Psi_{0} \mathrm{H} \alpha$ & $\Psi_{0}[\mathrm{~N} \mathrm{II}]$ \\
\hline $1-339094$ & 40.2 & $133.0 \pm 1.0$ & $160.0 \pm 1.0$ & $152.0 \pm 2.0$ & $157.0 \pm 2.0$ & $0.0 \pm 1.5$ & $176.0 \pm 1.8$ & $175.0 \pm 0.8$ & $8.0 \pm 0.5$ \\
\hline $1-274646$ & 38.91 & $121.0 \pm 1.0$ & $82.0 \pm 1.0$ & $108.0 \pm 1.0$ & $108.0 \pm 1.0$ & $61.5 \pm 17.2$ & $175.0 \pm 0.5$ & $41.0 \pm 0.5$ & $22.5 \pm 0.5$ \\
\hline $1-24099$ & 38.34 & $125.0 \pm 2.0$ & $84.0 \pm 2.0$ & $131.0 \pm 17.0$ & $153.0 \pm 6.0$ & $125.5 \pm 1.2$ & $135.5 \pm 0.8$ & $134.5 \pm 0.5$ & $121.5 \pm 0.5$ \\
\hline $1-137883$ & 40.15 & $185.0 \pm 5.0$ & $164.0 \pm 3.0$ & $133.0 \pm 3.0$ & $130.0 \pm 2.0$ & $55.5 \pm 2.0$ & $56.5 \pm 0.8$ & $48.0 \pm 1.2$ & $41.5 \pm 0.5$ \\
\hline $1-178838$ & 38.69 & $114.0 \pm 2.0$ & $90.0 \pm 5.0$ & $107.0 \pm 3.0$ & $103.0 \pm 2.0$ & $84.5 \pm 1.5$ & $44.0 \pm 0.5$ & $41.0 \pm 4.8$ & $36.0 \pm 2.2$ \\
\hline $1-36878$ & 39.07 & $138.0 \pm 3.0$ & $101.0 \pm 1.0$ & $90.0 \pm 1.0$ & $98.0 \pm 1.0$ & $46.0 \pm 1.0$ & $26.0 \pm 0.5$ & $43.0 \pm 0.5$ & $45.5 \pm 1.2$ \\
\hline $1-48116$ & 40.12 & $151.0 \pm 1.0$ & $178.0 \pm 1.0$ & $127.0 \pm 1.0$ & $134.0 \pm 1.0$ & $56.0 \pm 1.0$ & $65.0 \pm 0.5$ & $57.0 \pm 1.0$ & $56.0 \pm 0.5$ \\
\hline $1-386452$ & 39.09 & $123.0 \pm 1.0$ & $90.0 \pm 1.0$ & $89.0 \pm 1.0$ & $91.0 \pm 1.0$ & $135.0 \pm 2.0$ & $131.0 \pm 0.5$ & $131.5 \pm 1.0$ & $131.0 \pm 1.2$ \\
\hline $1-24416$ & 38.76 & $157.0 \pm 1.0$ & $104.0 \pm 1.0$ & $162.0 \pm 3.0$ & $148.0 \pm 2.0$ & $85.0 \pm 0.8$ & $79.5 \pm 0.8$ & $83.5 \pm 0.5$ & $82.5 \pm 0.5$ \\
\hline $1-256446$ & 40.12 & $214.0 \pm 2.0$ & $244.0 \pm 1.0$ & $231.0 \pm 1.0$ & $232.0 \pm 1.0$ & $158.0 \pm 11.0$ & $62.0 \pm 0.5$ & $48.0 \pm 4.2$ & $49.5 \pm 0.5$ \\
\hline $1-322671$ & 38.7 & $173.0 \pm 1.0$ & $134.0 \pm 12.0$ & $360.0 \pm 121.0$ & $167.0 \pm 12.0$ & $130.0 \pm 3.2$ & $109.0 \pm 0.5$ & $101.5 \pm 0.5$ & $143.0 \pm 0.5$ \\
\hline $1-256465$ & 39.38 & $217.0 \pm 1.0$ & $227.0 \pm 4.0$ & $187.0 \pm 3.0$ & $216.0 \pm 6.0$ & $134.0 \pm 16.0$ & $75.0 \pm 0.5$ & $62.5 \pm 0.5$ & $64.0 \pm 0.5$ \\
\hline $1-95585$ & 40.11 & $171.0 \pm 1.0$ & $214.0 \pm 3.0$ & $211.0 \pm 3.0$ & $206.0 \pm 2.0$ & $69.5 \pm 1.0$ & $62.5 \pm 0.5$ & $68.0 \pm 0.5$ & $69.5 \pm 0.5$ \\
\hline $1-166947$ & 38.79 & $149.0 \pm 1.0$ & $83.0 \pm 3.0$ & $143.0 \pm 3.0$ & $116.0 \pm 2.0$ & $36.0 \pm 3.2$ & $123.0 \pm 0.5$ & $34.0 \pm 0.8$ & $35.5 \pm 0.8$ \\
\hline $1-210593$ & 39.04 & $160.0 \pm 1.0$ & $128.0 \pm 3.0$ & $146.0 \pm 4.0$ & $147.0 \pm 4.0$ & $101.5 \pm 1.0$ & $107.5 \pm 0.5$ & $104.0 \pm 0.8$ & $103.0 \pm 0.5$ \\
\hline $1-135641$ & 40.04 & $165.0 \pm 4.0$ & $168.0 \pm 2.0$ & $155.0 \pm 3.0$ & $148.0 \pm 1.0$ & $164.5 \pm 0.5$ & $168.5 \pm 0.5$ & $163.5 \pm 0.5$ & $166.5 \pm 0.5$ \\
\hline $1-635503$ & 39.05 & $121.0 \pm 1.0$ & $72.0 \pm 1.0$ & $82.0 \pm 1.0$ & $83.0 \pm 1.0$ & $140.0 \pm 1.2$ & $145.0 \pm 0.5$ & $141.5 \pm 0.5$ & $138.5 \pm 0.5$ \\
\hline $1-235398$ & 38.74 & $154.0 \pm 1.0$ & $86.0 \pm 2.0$ & $123.0 \pm 1.0$ & $120.0 \pm 1.0$ & $98.0 \pm 0.5$ & $102.0 \pm 0.5$ & $99.5 \pm 0.5$ & $100.0 \pm 0.5$ \\
\hline $1-259142$ & 40.09 & $211.0 \pm 1.0$ & $272.0 \pm 5.0$ & $248.0 \pm 4.0$ & $274.0 \pm 4.0$ & $102.5 \pm 0.5$ & $100.0 \pm 0.5$ & $106.5 \pm 0.5$ & $101.5 \pm 0.5$ \\
\hline $1-55572$ & 38.93 & $194.0 \pm 2.0$ & $117.0 \pm 1.0$ & $208.0 \pm 4.0$ & $198.0 \pm 5.0$ & $51.0 \pm 0.5$ & $52.0 \pm 0.5$ & $50.5 \pm 0.5$ & $52.0 \pm 0.8$ \\
\hline $1-489649$ & 39.05 & $170.0 \pm 1.0$ & $100.0 \pm 2.0$ & $170.0 \pm 6.0$ & $155.0 \pm 2.0$ & $170.5 \pm 1.5$ & $169.0 \pm 0.5$ & $1.0 \pm 0.5$ & $171.5 \pm 0.8$ \\
\hline $1-109056$ & 40.07 & $124.0 \pm 1.0$ & $104.0 \pm 1.0$ & $104.0 \pm 1.0$ & $115.0 \pm 1.0$ & $157.0 \pm 1.5$ & $159.0 \pm 0.5$ & $156.0 \pm 1.0$ & $154.0 \pm 1.0$ \\
\hline $1-73005$ & 38.81 & $107.0 \pm 1.0$ & $91.0 \pm 4.0$ & $91.0 \pm 1.0$ & $91.0 \pm 1.0$ & $43.5 \pm 2.2$ & $59.5 \pm 0.5$ & $44.0 \pm 1.0$ & $44.0 \pm 1.2$ \\
\hline $1-43009$ & 38.65 & $94.0 \pm 1.0$ & $79.0 \pm 2.0$ & $83.0 \pm 1.0$ & $85.0 \pm 1.0$ & $168.0 \pm 1.2$ & $172.5 \pm 1.0$ & $171.0 \pm 1.0$ & $171.5 \pm 1.0$ \\
\hline $1-24148$ & 40.06 & $149.0 \pm 1.0$ & $126.0 \pm 1.0$ & $172.0 \pm 1.0$ & $159.0 \pm 1.0$ & $155.5 \pm 0.8$ & $150.5 \pm 0.5$ & $163.5 \pm 0.5$ & $134.5 \pm 0.5$ \\
\hline $1-285031$ & 38.95 & $117.0 \pm 1.0$ & $67.0 \pm 1.0$ & $83.0 \pm 0.0$ & $82.0 \pm 0.0$ & $38.0 \pm 0.8$ & $38.0 \pm 0.5$ & $37.0 \pm 0.8$ & $36.5 \pm 0.8$ \\
\hline $1-236099$ & 38.51 & $85.0 \pm 1.0$ & $96.0 \pm 3.0$ & $102.0 \pm 4.0$ & $83.0 \pm 4.0$ & $1.0 \pm 1.7$ & $138.0 \pm 2.2$ & $37.5 \pm 0.5$ & $118.5 \pm 0.5$ \\
\hline $1-166919$ & 39.63 & $164.0 \pm 2.0$ & $158.0 \pm 3.0$ & $153.0 \pm 4.0$ & $223.0 \pm 4.0$ & $105.0 \pm 1.0$ & $106.0 \pm 0.5$ & $103.0 \pm 0.5$ & $102.5 \pm 0.8$ \\
\hline $12-129446$ & 39.24 & $115.0 \pm 1.0$ & $113.0 \pm 3.0$ & $84.0 \pm 1.0$ & $97.0 \pm 2.0$ & $159.0 \pm 1.5$ & $161.0 \pm 0.5$ & $157.0 \pm 1.0$ & $157.5 \pm 0.8$ \\
\hline $1-90849$ & 39.1 & $115.0 \pm 1.0$ & $88.0 \pm 2.0$ & $81.0 \pm 1.0$ & $95.0 \pm 1.0$ & $58.5 \pm 1.0$ & $51.5 \pm 0.5$ & $55.5 \pm 0.5$ & $57.5 \pm 0.5$ \\
\hline $1-248389$ & 39.91 & $148.0 \pm 0.0$ & $144.0 \pm 1.0$ & $175.0 \pm 1.0$ & $160.0 \pm 1.0$ & $177.0 \pm 1.5$ & $145.0 \pm 0.5$ & $128.5 \pm 0.5$ & $145.5 \pm 0.5$ \\
\hline $1-94554$ & 38.98 & $136.0 \pm 1.0$ & $111.0 \pm 3.0$ & $135.0 \pm 2.0$ & $146.0 \pm 5.0$ & $29.5 \pm 2.0$ & $37.5 \pm 0.5$ & $8.0 \pm 0.8$ & $44.0 \pm 0.5$ \\
\hline $1-245774$ & 38.97 & $130.0 \pm 1.0$ & $99.0 \pm 2.0$ & $98.0 \pm 1.0$ & $107.0 \pm 1.0$ & $85.5 \pm 1.2$ & $91.5 \pm 0.5$ & $85.5 \pm 0.8$ & $87.0 \pm 0.5$ \\
\hline $1-321739$ & 39.9 & $172.0 \pm 4.0$ & $145.0 \pm 2.0$ & $109.0 \pm 1.0$ & $123.0 \pm 1.0$ & $172.0 \pm 0.5$ & $163.5 \pm 0.5$ & $174.0 \pm 0.5$ & $179.0 \pm 0.5$ \\
\hline $1-247417$ & 38.77 & $107.0 \pm 1.0$ & $82.0 \pm 2.0$ & $85.0 \pm 1.0$ & $90.0 \pm 1.0$ & $142.0 \pm 0.5$ & $141.0 \pm 0.5$ & $141.0 \pm 0.5$ & $141.5 \pm 0.5$ \\
\hline $1-633994$ & 38.79 & $238.0 \pm 26.0$ & $99.0 \pm 2.0$ & $131.0 \pm 4.0$ & $201.0 \pm 21.0$ & $124.5 \pm 0.8$ & $135.5 \pm 0.5$ & $123.0 \pm 1.0$ & $129.5 \pm 0.5$ \\
\hline $1-234618$ & 39.99 & $182.0 \pm 17.0$ & $167.0 \pm 3.0$ & $123.0 \pm 9.0$ & $137.0 \pm 1.0$ & $23.0 \pm 0.5$ & $16.0 \pm 1.2$ & $19.0 \pm 0.5$ & $23.0 \pm 0.8$ \\
\hline $1-282144$ & 39.16 & $83.0 \pm 1.0$ & $93.0 \pm 2.0$ & $70.0 \pm 1.0$ & $75.0 \pm 1.0$ & $179.0 \pm 2.5$ & $163.0 \pm 0.5$ & $179.0 \pm 1.5$ & $165.5 \pm 0.5$ \\
\hline $1-339125$ & 38.97 & $181.0 \pm 2.0$ & $132.0 \pm 3.0$ & $284.0 \pm 44.0$ & $653.0 \pm 231.0$ & $170.5 \pm 1.5$ & $179.5 \pm 0.5$ & $141.0 \pm 0.5$ & $165.0 \pm 0.5$ \\
\hline $1-229010$ & 39.88 & $224.0 \pm 1.0$ & $237.0 \pm 2.0$ & $268.0 \pm 4.0$ & $228.0 \pm 1.0$ & $177.5 \pm 0.5$ & $1.5 \pm 0.5$ & $178.0 \pm 0.5$ & $0.5 \pm 0.5$ \\
\hline $1-210962$ & 39.01 & $194.0 \pm 1.0$ & $117.0 \pm 2.0$ & $147.0 \pm 2.0$ & $157.0 \pm 2.0$ & $91.5 \pm 1.2$ & $96.5 \pm 0.5$ & $89.0 \pm 0.5$ & $89.5 \pm 0.5$ \\
\hline $1-613211$ & 38.65 & $242.0 \pm 1.0$ & $108.0 \pm 3.0$ & $192.0 \pm 3.0$ & $188.0 \pm 4.0$ & $22.0 \pm 0.5$ & $16.5 \pm 0.5$ & $18.0 \pm 0.5$ & $22.5 \pm 0.5$ \\
\hline $1-211311$ & 39.83 & $114.0 \pm 1.0$ & $173.0 \pm 2.0$ & $191.0 \pm 2.0$ & $219.0 \pm 3.0$ & $160.0 \pm 3.0$ & $156.0 \pm 0.5$ & $167.5 \pm 2.5$ & $166.0 \pm 2.0$ \\
\hline $1-25688$ & 38.35 & $110.0 \pm 1.0$ & $64.0 \pm 2.0$ & $110.0 \pm 2.0$ & $115.0 \pm 1.0$ & $20.5 \pm 1.5$ & $13.5 \pm 0.5$ & $17.0 \pm 0.8$ & $19.5 \pm 1.5$ \\
\hline $1-94422$ & 38.87 & $129.0 \pm 1.0$ & $82.0 \pm 1.0$ & $131.0 \pm 2.0$ & $123.0 \pm 1.0$ & $47.0 \pm 1.0$ & $28.0 \pm 0.5$ & $49.0 \pm 1.0$ & $52.0 \pm 0.8$ \\
\hline $1-373161$ & 39.86 & $211.0 \pm 0.0$ & $139.0 \pm 1.0$ & $140.0 \pm 1.0$ & $135.0 \pm 1.0$ & $124.0 \pm 0.5$ & $164.5 \pm 0.5$ & $126.0 \pm 0.5$ & $33.5 \pm 0.5$ \\
\hline $1-259650$ & 39.47 & $224.0 \pm 1.0$ & $234.0 \pm 5.0$ & $161.0 \pm 5.0$ & $209.0 \pm 50.0$ & $166.5 \pm 0.8$ & $170.5 \pm 0.5$ & $158.5 \pm 0.5$ & $166.0 \pm 0.5$ \\
\hline $1-289865$ & 38.73 & $271.0 \pm 2.0$ & $128.0 \pm 10.0$ & $313.0 \pm 13.0$ & $213.0 \pm 10.0$ & $40.0 \pm 1.8$ & $97.5 \pm 0.5$ & $58.5 \pm 0.5$ & $22.5 \pm 0.5$ \\
\hline
\end{tabular}


Table A1 - continued

\begin{tabular}{|c|c|c|c|c|c|c|c|c|c|}
\hline mangaid & $\log _{10} L_{[} \mathrm{O} \mathrm{III]}$ & $\sigma_{\star}$ & $\sigma_{[[\mathrm{O} \mathrm{III}]}$ & $\sigma_{H \alpha}$ & $\left.\sigma_{[\mathrm{N}} \mathrm{II}\right]$ & $\Psi_{0 \star}$ & $\Psi_{0}[\mathrm{O} \mathrm{III}]$ & $\Psi_{0} \mathrm{H} \alpha$ & $\Psi_{0}\left[\mathrm{~N}_{\mathrm{II}}\right]$ \\
\hline $1-210646$ & 39.84 & $96.0 \pm 1.0$ & $108.0 \pm 1.0$ & $91.0 \pm 1.0$ & $104.0 \pm 1.0$ & $106.5 \pm 0.8$ & $107.0 \pm 1.0$ & $106.5 \pm 0.5$ & $106.5 \pm 0.8$ \\
\hline $1-114306$ & 38.65 & $101.0 \pm 1.0$ & $44.0 \pm 3.0$ & $95.0 \pm 1.0$ & $113.0 \pm 3.0$ & $128.5 \pm 2.0$ & $135.0 \pm 0.8$ & $134.0 \pm 0.8$ & $133.5 \pm 0.8$ \\
\hline $1-487130$ & 38.93 & $75.0 \pm 1.0$ & $50.0 \pm 2.0$ & $69.0 \pm 1.0$ & $84.0 \pm 2.0$ & $97.0 \pm 1.5$ & $98.0 \pm 0.5$ & $90.5 \pm 0.5$ & $95.5 \pm 0.5$ \\
\hline $1-351790$ & 39.83 & $82.0 \pm 1.0$ & $88.0 \pm 1.0$ & $83.0 \pm 1.0$ & $82.0 \pm 2.0$ & $135.5 \pm 2.2$ & $96.5 \pm 1.8$ & $83.5 \pm 2.2$ & $91.5 \pm 0.8$ \\
\hline $1-23731$ & 37.83 & $140.0 \pm 1.0$ & $106.0 \pm 7.0$ & $264.0 \pm 16.0$ & $406.0 \pm 54.0$ & $134.0 \pm 2.0$ & $145.5 \pm 0.8$ & $108.0 \pm 0.5$ & $126.5 \pm 0.5$ \\
\hline $1-167334$ & 38.83 & $111.0 \pm 1.0$ & $89.0 \pm 1.0$ & $136.0 \pm 1.0$ & $138.0 \pm 1.0$ & $120.5 \pm 2.0$ & $166.5 \pm 0.5$ & $11.0 \pm 34.5$ & $56.0 \pm 4.5$ \\
\hline $1-163831$ & 39.64 & $138.0 \pm 1.0$ & $173.0 \pm 3.0$ & $157.0 \pm 4.0$ & $209.0 \pm 3.0$ & $95.5 \pm 1.2$ & $93.0 \pm 0.5$ & $95.5 \pm 1.2$ & $97.0 \pm 0.5$ \\
\hline $1-247456$ & 39.3 & $879.0 \pm 34.0$ & $172.0 \pm 13.0$ & $1090.0 \pm 327.0$ & $749.0 \pm 91.0$ & $149.0 \pm 1.2$ & $169.0 \pm 0.5$ & $167.5 \pm 0.8$ & $167.5 \pm 0.5$ \\
\hline $1-210593$ & 39.04 & $160.0 \pm 1.0$ & $128.0 \pm 3.0$ & $146.0 \pm 4.0$ & $147.0 \pm 4.0$ & $101.5 \pm 1.0$ & $107.5 \pm 0.5$ & $104.0 \pm 0.8$ & $103.0 \pm 0.5$ \\
\hline $1-22301$ & 39.84 & $163.0 \pm 2.0$ & $177.0 \pm 4.0$ & $137.0 \pm 5.0$ & $177.0 \pm 5.0$ & $9.5 \pm 1.5$ & $8.5 \pm 1.8$ & $4.5 \pm 0.5$ & $4.0 \pm 0.5$ \\
\hline $1-251871$ & 39.15 & $160.0 \pm 2.0$ & $108.0 \pm 5.0$ & $128.0 \pm 2.0$ & $127.0 \pm 3.0$ & $63.0 \pm 0.5$ & $66.0 \pm 0.5$ & $63.0 \pm 0.5$ & $61.5 \pm 1.0$ \\
\hline $1-72914$ & 39.01 & $143.0 \pm 1.0$ & $101.0 \pm 4.0$ & $110.0 \pm 2.0$ & $136.0 \pm 4.0$ & $72.5 \pm 0.8$ & $82.5 \pm 0.8$ & $76.0 \pm 0.5$ & $74.0 \pm 0.5$ \\
\hline $1-248420$ & 39.71 & $138.0 \pm 1.0$ & $159.0 \pm 1.0$ & $152.0 \pm 2.0$ & $151.0 \pm 2.0$ & $49.5 \pm 1.0$ & $49.5 \pm 1.0$ & $49.0 \pm 1.0$ & $49.0 \pm 1.0$ \\
\hline $1-211063$ & 38.75 & $136.0 \pm 1.0$ & $76.0 \pm 2.0$ & $141.0 \pm 2.0$ & $111.0 \pm 2.0$ & $170.0 \pm 1.8$ & $164.0 \pm 0.5$ & $166.5 \pm 1.8$ & $165.0 \pm 1.2$ \\
\hline $1-211074$ & 38.86 & $154.0 \pm 0.0$ & $126.0 \pm 2.0$ & $150.0 \pm 2.0$ & $138.0 \pm 2.0$ & $142.5 \pm 1.0$ & $133.5 \pm 0.5$ & $142.5 \pm 0.8$ & $143.0 \pm 0.8$ \\
\hline $1-23979$ & 39.72 & $118.0 \pm 1.0$ & $132.0 \pm 1.0$ & $115.0 \pm 1.0$ & $120.0 \pm 1.0$ & $56.5 \pm 1.8$ & $67.5 \pm 0.5$ & $75.5 \pm 0.5$ & $59.0 \pm 0.5$ \\
\hline $1-320681$ & 37.49 & $189.0 \pm 2.0$ & $104.0 \pm 22.0$ & $288.0 \pm 14.0$ & $306.0 \pm 31.0$ & $53.0 \pm 0.8$ & $67.0 \pm 1.0$ & $53.0 \pm 9.8$ & $33.0 \pm 0.5$ \\
\hline $1-519738$ & 38.44 & $158.0 \pm 1.0$ & $101.0 \pm 3.0$ & $135.0 \pm 7.0$ & $237.0 \pm 19.0$ & $56.5 \pm 1.0$ & $52.5 \pm 0.5$ & $60.0 \pm 0.5$ & $52.5 \pm 0.5$ \\
\hline $1-542318$ & 39.88 & $143.0 \pm 1.0$ & $144.0 \pm 2.0$ & $172.0 \pm 2.0$ & $164.0 \pm 2.0$ & $71.0 \pm 2.8$ & $119.0 \pm 0.5$ & $82.0 \pm 0.5$ & $96.0 \pm 0.5$ \\
\hline $1-285052$ & 38.79 & $143.0 \pm 1.0$ & $114.0 \pm 4.0$ & $137.0 \pm 2.0$ & $110.0 \pm 1.0$ & $98.0 \pm 2.2$ & $75.0 \pm 0.5$ & $93.5 \pm 1.2$ & $97.0 \pm 1.5$ \\
\hline $1-377125$ & 38.98 & $143.0 \pm 1.0$ & $99.0 \pm 2.0$ & $144.0 \pm 4.0$ & $107.0 \pm 3.0$ & $136.5 \pm 9.0$ & $100.5 \pm 0.5$ & $129.0 \pm 0.8$ & $129.0 \pm 0.5$ \\
\hline $1-95092$ & 39.75 & $143.0 \pm 1.0$ & $154.0 \pm 3.0$ & $130.0 \pm 2.0$ & $154.0 \pm 2.0$ & $85.5 \pm 1.0$ & $80.5 \pm 0.5$ & $86.0 \pm 0.5$ & $85.5 \pm 0.5$ \\
\hline $1-210962$ & 39.01 & $194.0 \pm 1.0$ & $117.0 \pm 2.0$ & $147.0 \pm 2.0$ & $157.0 \pm 2.0$ & $91.5 \pm 1.2$ & $96.5 \pm 0.5$ & $89.0 \pm 0.5$ & $89.5 \pm 0.5$ \\
\hline $1-251279$ & 39.15 & $156.0 \pm 1.0$ & $120.0 \pm 2.0$ & $117.0 \pm 2.0$ & $130.0 \pm 2.0$ & $150.0 \pm 1.0$ & $142.5 \pm 0.5$ & $146.0 \pm 0.5$ & $146.0 \pm 0.5$ \\
\hline $1-279676$ & 39.54 & $155.0 \pm 1.0$ & $125.0 \pm 2.0$ & $120.0 \pm 2.0$ & $145.0 \pm 2.0$ & $20.0 \pm 1.5$ & $27.5 \pm 0.5$ & $19.5 \pm 0.5$ & $19.5 \pm 0.5$ \\
\hline $1-44789$ & 39.1 & $154.0 \pm 2.0$ & $142.0 \pm 3.0$ & $173.0 \pm 4.0$ & $148.0 \pm 1.0$ & $85.0 \pm 0.8$ & $95.0 \pm 0.5$ & $88.0 \pm 1.5$ & $90.0 \pm 0.5$ \\
\hline $1-378401$ & 39.38 & $224.0 \pm 2.0$ & $223.0 \pm 4.0$ & $188.0 \pm 7.0$ & $232.0 \pm 11.0$ & $162.5 \pm 1.0$ & $33.5 \pm 0.5$ & $38.0 \pm 0.5$ & $26.5 \pm 0.5$ \\
\hline $1-201561$ & 39.59 & $168.0 \pm 2.0$ & $157.0 \pm 2.0$ & $168.0 \pm 2.0$ & $166.0 \pm 1.0$ & $23.5 \pm 3.0$ & $22.5 \pm 0.5$ & $29.5 \pm 0.5$ & $42.5 \pm 0.5$ \\
\hline $1-24246$ & 39.1 & $110.0 \pm 1.0$ & $134.0 \pm 4.0$ & $94.0 \pm 2.0$ & $105.0 \pm 2.0$ & $139.0 \pm 1.5$ & $134.0 \pm 0.8$ & $134.0 \pm 1.0$ & $121.5 \pm 0.5$ \\
\hline $1-285052$ & 38.79 & $143.0 \pm 1.0$ & $114.0 \pm 4.0$ & $137.0 \pm 2.0$ & $110.0 \pm 1.0$ & $98.0 \pm 2.2$ & $75.0 \pm 0.5$ & $93.5 \pm 1.2$ & $97.0 \pm 1.5$ \\
\hline 1-198182 & 39.68 & $217.0 \pm 1.0$ & $195.0 \pm 1.0$ & $219.0 \pm 1.0$ & $227.0 \pm 2.0$ & $44.5 \pm 0.8$ & $35.5 \pm 0.8$ & $42.5 \pm 0.8$ & $55.0 \pm 0.5$ \\
\hline $1-256185$ & 39.22 & $214.0 \pm 1.0$ & $171.0 \pm 10.0$ & $151.0 \pm 5.0$ & $202.0 \pm 12.0$ & $178.5 \pm 0.8$ & $162.5 \pm 0.8$ & $167.5 \pm 0.8$ & $168.0 \pm 1.2$ \\
\hline $1-48053$ & - & $286.0 \pm 1.0$ & $116.0 \pm 36.0$ & $184.0 \pm 10.0$ & $294.0 \pm 13.0$ & $12.0 \pm 0.8$ & $161.5 \pm 0.5$ & $39.0 \pm 0.5$ & $160.0 \pm 0.5$ \\
\hline $1-96075$ & 39.53 & $122.0 \pm 1.0$ & $133.0 \pm 2.0$ & $115.0 \pm 2.0$ & $137.0 \pm 2.0$ & $42.0 \pm 0.8$ & $47.5 \pm 0.5$ & $43.5 \pm 0.5$ & $44.0 \pm 0.5$ \\
\hline $1-166947$ & 38.79 & $149.0 \pm 1.0$ & $83.0 \pm 3.0$ & $143.0 \pm 3.0$ & $116.0 \pm 2.0$ & $36.0 \pm 3.2$ & $123.0 \pm 0.5$ & $34.0 \pm 0.8$ & $35.5 \pm 0.8$ \\
\hline $1-52259$ & 38.98 & $89.0 \pm 1.0$ & $100.0 \pm 3.0$ & $63.0 \pm 0.0$ & $63.0 \pm 0.0$ & $90.5 \pm 4.2$ & $103.0 \pm 0.5$ & $87.5 \pm 12.0$ & $99.5 \pm 6.2$ \\
\hline $1-519742$ & 39.78 & $96.0 \pm 1.0$ & $99.0 \pm 0.0$ & $86.0 \pm 1.0$ & $80.0 \pm 1.0$ & $133.0 \pm 4.0$ & $124.5 \pm 0.5$ & $129.5 \pm 1.8$ & $120.5 \pm 1.0$ \\
\hline $1-37079$ & 37.71 & $72.0 \pm 1.0$ & $39.0 \pm 3.0$ & $78.0 \pm 1.0$ & $96.0 \pm 2.0$ & $157.5 \pm 6.0$ & $15.0 \pm 0.5$ & $177.5 \pm 3.0$ & $25.5 \pm 0.5$ \\
\hline $1-276679$ & 38.42 & $88.0 \pm 1.0$ & $99.0 \pm 4.0$ & $61.0 \pm 0.0$ & $71.0 \pm 0.0$ & $152.0 \pm 2.2$ & $137.5 \pm 0.5$ & $151.5 \pm 0.8$ & $152.5 \pm 0.5$ \\
\hline $1-491229$ & 39.64 & $189.0 \pm 1.0$ & $213.0 \pm 5.0$ & $249.0 \pm 3.0$ & $226.0 \pm 2.0$ & $101.5 \pm 1.2$ & $82.5 \pm 0.5$ & $99.5 \pm 0.5$ & $105.0 \pm 0.5$ \\
\hline $1-94554$ & 38.98 & $136.0 \pm 1.0$ & $111.0 \pm 3.0$ & $135.0 \pm 2.0$ & $146.0 \pm 5.0$ & $29.5 \pm 2.0$ & $37.5 \pm 0.5$ & $8.0 \pm 0.8$ & $44.0 \pm 0.5$ \\
\hline $1-604048$ & 39.14 & $163.0 \pm 1.0$ & $126.0 \pm 1.0$ & $124.0 \pm 2.0$ & $129.0 \pm 3.0$ & $58.5 \pm 2.5$ & $71.0 \pm 0.5$ & $59.0 \pm 1.8$ & $67.0 \pm 1.8$ \\
\hline $1-604761$ & 39.64 & $193.0 \pm 2.0$ & $186.0 \pm 3.0$ & $178.0 \pm 2.0$ & $189.0 \pm 3.0$ & $70.0 \pm 0.5$ & $68.0 \pm 0.5$ & $66.5 \pm 0.5$ & $66.5 \pm 0.5$ \\
\hline $1-210173$ & 39.28 & $155.0 \pm 2.0$ & $137.0 \pm 4.0$ & $113.0 \pm 2.0$ & $135.0 \pm 3.0$ & $122.5 \pm 1.0$ & $121.0 \pm 0.5$ & $121.0 \pm 0.5$ & $124.5 \pm 0.5$ \\
\hline $1-71525$ & 39.0 & $136.0 \pm 1.0$ & $94.0 \pm 5.0$ & $242.0 \pm 135.0$ & $685.0 \pm 263.0$ & $126.5 \pm 1.2$ & $138.0 \pm 0.5$ & $130.5 \pm 0.8$ & $130.5 \pm 0.5$ \\
\hline $1-25725$ & 39.39 & $190.0 \pm 2.0$ & $198.0 \pm 3.0$ & $195.0 \pm 4.0$ & $225.0 \pm 4.0$ & $138.5 \pm 2.2$ & $136.5 \pm 0.5$ & $133.0 \pm 1.2$ & $144.5 \pm 0.5$ \\
\hline $1-211079$ & 38.53 & $198.0 \pm 1.0$ & $228.0 \pm 11.0$ & $250.0 \pm 18.0$ & $252.0 \pm 15.0$ & $178.5 \pm 0.8$ & $167.0 \pm 0.5$ & $126.5 \pm 0.5$ & $125.5 \pm 0.5$ \\
\hline $1-322074$ & 38.12 & $120.0 \pm 0.0$ & $93.0 \pm 3.0$ & $227.0 \pm 13.0$ & $234.0 \pm 49.0$ & $35.5 \pm 2.5$ & $68.5 \pm 0.5$ & $120.0 \pm 0.5$ & $160.5 \pm 0.5$ \\
\hline
\end{tabular}


Table A1 - continued

\begin{tabular}{|c|c|c|c|c|c|c|c|c|c|}
\hline mangaid & $\left.\log _{10} L_{[\mathrm{O}} \mathrm{III}\right]$ & $\sigma_{\star}$ & $\sigma_{[} \mathrm{O}$ III $]$ & $\sigma_{H \alpha}$ & $\left.\sigma_{[\mathrm{N}} \mathrm{II}\right]$ & $\Psi_{0 \star}$ & $\Psi_{0}[\mathrm{O} \mathrm{III}]$ & $\Psi_{0} \mathrm{H} \alpha$ & $\Psi_{0}[\mathrm{~N}$ II $]$ \\
\hline $1-94604$ & 39.5 & $110.0 \pm 1.0$ & $109.0 \pm 1.0$ & $136.0 \pm 4.0$ & $343.0 \pm 54.0$ & $153.0 \pm 2.0$ & $166.0 \pm 0.5$ & $157.5 \pm 0.5$ & $144.0 \pm 0.5$ \\
\hline $1-295095$ & 38.76 & $101.0 \pm 1.0$ & $112.0 \pm 3.0$ & $80.0 \pm 1.0$ & $88.0 \pm 2.0$ & $1.0 \pm 2.2$ & $3.0 \pm 0.8$ & $178.0 \pm 1.5$ & $1.0 \pm 1.2$ \\
\hline $1-134239$ & 39.17 & $138.0 \pm 1.0$ & $136.0 \pm 3.0$ & $119.0 \pm 3.0$ & $140.0 \pm 3.0$ & $32.5 \pm 1.5$ & $31.5 \pm 0.5$ & $34.0 \pm 1.5$ & $35.5 \pm 1.5$ \\
\hline $1-37036$ & 39.42 & $220.0 \pm 1.0$ & $173.0 \pm 2.0$ & $206.0 \pm 2.0$ & $197.0 \pm 3.0$ & $98.0 \pm 0.8$ & $86.0 \pm 0.5$ & $84.0 \pm 0.5$ & $99.0 \pm 0.5$ \\
\hline $1-210785$ & 37.08 & $165.0 \pm 1.0$ & $102.0 \pm 12.0$ & $569.0 \pm 171.0$ & $2128.0 \pm 444.0$ & $125.5 \pm 0.8$ & $130.0 \pm 0.5$ & $113.5 \pm 0.5$ & $107.0 \pm 0.5$ \\
\hline $1-25680$ & 39.08 & $230.0 \pm 1.0$ & $140.0 \pm 3.0$ & $176.0 \pm 3.0$ & $189.0 \pm 4.0$ & $55.5 \pm 2.0$ & $168.0 \pm 0.5$ & $1.0 \pm 0.5$ & $174.5 \pm 0.5$ \\
\hline $1-167688$ & 39.56 & $96.0 \pm 1.0$ & $81.0 \pm 0.0$ & $95.0 \pm 0.0$ & $89.0 \pm 1.0$ & $35.5 \pm 9.2$ & $18.0 \pm 1.0$ & $40.0 \pm 0.8$ & $70.5 \pm 1.5$ \\
\hline $1-235587$ & 38.59 & $125.0 \pm 0.0$ & $84.0 \pm 1.0$ & $105.0 \pm 2.0$ & $106.0 \pm 2.0$ & $85.5 \pm 2.2$ & $92.0 \pm 0.5$ & $99.0 \pm 1.8$ & $100.0 \pm 1.0$ \\
\hline $1-37062$ & 39.02 & $79.0 \pm 1.0$ & $83.0 \pm 1.0$ & $91.0 \pm 1.0$ & $96.0 \pm 1.0$ & $21.5 \pm 3.5$ & $173.0 \pm 0.5$ & $19.0 \pm 12.0$ & $31.0 \pm 0.5$ \\
\hline $1-279666$ & 39.45 & $115.0 \pm 1.0$ & $103.0 \pm 1.0$ & $104.0 \pm 1.0$ & $121.0 \pm 1.0$ & $57.5 \pm 2.2$ & $52.0 \pm 0.5$ & $60.0 \pm 1.8$ & $46.5 \pm 1.5$ \\
\hline $1-392976$ & 38.57 & $108.0 \pm 1.0$ & $94.0 \pm 3.0$ & $133.0 \pm 5.0$ & $123.0 \pm 4.0$ & $16.0 \pm 0.5$ & $56.0 \pm 0.5$ & $80.5 \pm 0.5$ & $166.0 \pm 0.5$ \\
\hline $1-47499$ & 38.86 & $110.0 \pm 0.0$ & $102.0 \pm 3.0$ & $128.0 \pm 4.0$ & $121.0 \pm 4.0$ & $108.0 \pm 2.2$ & $82.5 \pm 1.8$ & $108.0 \pm 0.5$ & $126.0 \pm 0.5$ \\
\hline $1-339163$ & 39.35 & $159.0 \pm 1.0$ & $323.0 \pm 6.0$ & $245.0 \pm 4.0$ & $353.0 \pm 8.0$ & $38.5 \pm 0.5$ & $42.0 \pm 0.5$ & $39.0 \pm 0.5$ & $39.0 \pm 0.5$ \\
\hline $1-136125$ & 38.56 & $109.0 \pm 1.0$ & $61.0 \pm 2.0$ & $118.0 \pm 2.0$ & $112.0 \pm 2.0$ & $68.0 \pm 0.5$ & $69.0 \pm 0.5$ & $67.5 \pm 0.5$ & $67.5 \pm 0.5$ \\
\hline $1-626830$ & 38.62 & $137.0 \pm 1.0$ & $107.0 \pm 3.0$ & $115.0 \pm 2.0$ & $136.0 \pm 2.0$ & $22.5 \pm 0.5$ & $29.5 \pm 0.5$ & $22.5 \pm 0.5$ & $19.5 \pm 0.5$ \\
\hline $1-258774$ & 39.4 & $143.0 \pm 0.0$ & $114.0 \pm 2.0$ & $110.0 \pm 1.0$ & $135.0 \pm 0.0$ & $154.0 \pm 4.2$ & $152.0 \pm 1.2$ & $155.0 \pm 3.2$ & $172.5 \pm 3.8$ \\
\hline $1-379660$ & 39.12 & $139.0 \pm 1.0$ & $132.0 \pm 3.0$ & $118.0 \pm 4.0$ & $127.0 \pm 4.0$ & $115.5 \pm 1.2$ & $106.0 \pm 0.5$ & $111.5 \pm 0.8$ & $113.5 \pm 0.5$ \\
\hline $1-48208$ & 38.7 & $162.0 \pm 1.0$ & $94.0 \pm 2.0$ & $140.0 \pm 4.0$ & $91.0 \pm 5.0$ & $71.5 \pm 2.2$ & $75.0 \pm 0.5$ & $76.0 \pm 0.5$ & $66.5 \pm 0.5$ \\
\hline $1-198153$ & 39.29 & $176.0 \pm 1.0$ & $145.0 \pm 2.0$ & $177.0 \pm 1.0$ & $178.0 \pm 1.0$ & $93.5 \pm 0.5$ & $96.0 \pm 0.5$ & $94.5 \pm 0.5$ & $94.5 \pm 0.5$ \\
\hline $1-211063$ & 38.75 & $136.0 \pm 1.0$ & $76.0 \pm 2.0$ & $141.0 \pm 2.0$ & $111.0 \pm 2.0$ & $170.0 \pm 1.8$ & $164.0 \pm 0.5$ & $166.5 \pm 1.8$ & $165.0 \pm 1.2$ \\
\hline $1-135810$ & 38.45 & $112.0 \pm 1.0$ & $80.0 \pm 2.0$ & $95.0 \pm 2.0$ & $101.0 \pm 2.0$ & $52.0 \pm 0.8$ & $54.5 \pm 0.5$ & $50.5 \pm 0.8$ & $50.5 \pm 0.5$ \\
\hline $1-91016$ & 39.49 & $103.0 \pm 1.0$ & $125.0 \pm 2.0$ & $138.0 \pm 3.0$ & $173.0 \pm 4.0$ & $50.5 \pm 4.0$ & $44.5 \pm 1.2$ & $51.5 \pm 1.2$ & $57.0 \pm 2.0$ \\
\hline $1-338828$ & 39.3 & $94.0 \pm 1.0$ & $90.0 \pm 1.0$ & $80.0 \pm 1.0$ & $80.0 \pm 1.0$ & $103.0 \pm 0.5$ & $101.5 \pm 0.5$ & $96.0 \pm 0.5$ & $86.0 \pm 1.2$ \\
\hline $1-386695$ & 39.68 & $105.0 \pm 2.0$ & $119.0 \pm 1.0$ & $105.0 \pm 1.0$ & $107.0 \pm 1.0$ & $129.0 \pm 1.2$ & $88.0 \pm 0.5$ & $141.5 \pm 0.5$ & $89.5 \pm 0.5$ \\
\hline $1-279073$ & 39.34 & $231.0 \pm 1.0$ & $161.0 \pm 4.0$ & $186.0 \pm 2.0$ & $181.0 \pm 3.0$ & $15.0 \pm 1.5$ & $169.5 \pm 0.8$ & $155.5 \pm 0.5$ & $164.0 \pm 0.5$ \\
\hline $1-211100$ & 38.09 & $198.0 \pm 1.0$ & $116.0 \pm 7.0$ & $275.0 \pm 44.0$ & $346.0 \pm 14.0$ & $152.0 \pm 4.8$ & $127.0 \pm 0.5$ & $103.0 \pm 0.5$ & $165.0 \pm 0.5$ \\
\hline $1-210784$ & 38.3 & $171.0 \pm 0.0$ & $97.0 \pm 4.0$ & $159.0 \pm 2.0$ & $218.0 \pm 3.0$ & $90.0 \pm 2.2$ & $101.0 \pm 0.5$ & $59.0 \pm 1.2$ & $62.5 \pm 0.5$ \\
\hline $1-135044$ & 39.44 & $115.0 \pm 1.0$ & $116.0 \pm 2.0$ & $156.0 \pm 2.0$ & $167.0 \pm 3.0$ & $95.5 \pm 1.2$ & $96.5 \pm 0.5$ & $96.5 \pm 1.2$ & $98.5 \pm 0.5$ \\
\hline $1-218280$ & 38.7 & $148.0 \pm 0.0$ & $104.0 \pm 1.0$ & $121.0 \pm 1.0$ & $114.0 \pm 3.0$ & $99.0 \pm 1.0$ & $96.0 \pm 0.5$ & $98.0 \pm 0.5$ & $98.0 \pm 0.5$ \\
\hline $1-211063$ & 38.75 & $136.0 \pm 1.0$ & $76.0 \pm 2.0$ & $141.0 \pm 2.0$ & $111.0 \pm 2.0$ & $170.0 \pm 1.8$ & $164.0 \pm 0.5$ & $166.5 \pm 1.8$ & $165.0 \pm 1.2$ \\
\hline $1-148068$ & 39.21 & $156.0 \pm 1.0$ & $121.0 \pm 4.0$ & $169.0 \pm 2.0$ & $156.0 \pm 1.0$ & $63.5 \pm 0.8$ & $59.0 \pm 0.5$ & $65.0 \pm 0.8$ & $66.5 \pm 0.5$ \\
\hline $1-166947$ & 38.79 & $149.0 \pm 1.0$ & $83.0 \pm 3.0$ & $143.0 \pm 3.0$ & $116.0 \pm 2.0$ & $36.0 \pm 3.2$ & $123.0 \pm 0.5$ & $34.0 \pm 0.8$ & $35.5 \pm 0.8$ \\
\hline $1-55572$ & 38.93 & $194.0 \pm 2.0$ & $117.0 \pm 1.0$ & $208.0 \pm 4.0$ & $198.0 \pm 5.0$ & $51.0 \pm 0.5$ & $52.0 \pm 0.5$ & $50.5 \pm 0.5$ & $52.0 \pm 0.8$ \\
\hline $1-277552$ & 39.27 & $118.0 \pm 1.0$ & $119.0 \pm 1.0$ & $99.0 \pm 1.0$ & $112.0 \pm 1.0$ & $3.5 \pm 0.5$ & $4.0 \pm 0.5$ & $2.5 \pm 0.5$ & $2.5 \pm 0.8$ \\
\hline $1-264513$ & 39.28 & $99.0 \pm 1.0$ & $106.0 \pm 1.0$ & $77.0 \pm 0.0$ & $85.0 \pm 1.0$ & $0.5 \pm 0.5$ & $177.0 \pm 3.8$ & $175.5 \pm 1.5$ & $175.5 \pm 2.0$ \\
\hline $1-136125$ & 38.56 & $109.0 \pm 1.0$ & $61.0 \pm 2.0$ & $118.0 \pm 2.0$ & $112.0 \pm 2.0$ & $68.0 \pm 0.5$ & $69.0 \pm 0.5$ & $67.5 \pm 0.5$ & $67.5 \pm 0.5$ \\
\hline $1-217050$ & 39.05 & $203.0 \pm 1.0$ & $154.0 \pm 2.0$ & $200.0 \pm 2.0$ & $208.0 \pm 3.0$ & $60.5 \pm 0.5$ & $83.0 \pm 0.5$ & $82.0 \pm 0.5$ & $78.0 \pm 0.5$ \\
\hline $1-135372$ & 38.66 & $212.0 \pm 1.0$ & $183.0 \pm 6.0$ & $1011.0 \pm 363.0$ & $280.0 \pm 13.0$ & $20.0 \pm 0.8$ & $23.0 \pm 0.5$ & $22.5 \pm 0.5$ & $29.5 \pm 0.5$ \\
\hline $1-274663$ & 38.87 & $216.0 \pm 1.0$ & $125.0 \pm 3.0$ & $188.0 \pm 3.0$ & $188.0 \pm 5.0$ & $153.5 \pm 1.8$ & $163.5 \pm 0.5$ & $111.5 \pm 0.5$ & $155.5 \pm 0.5$ \\
\hline $1-25554$ & 38.96 & $114.0 \pm 0.0$ & $111.0 \pm 1.0$ & $117.0 \pm 1.0$ & $112.0 \pm 2.0$ & $74.5 \pm 1.2$ & $67.5 \pm 0.5$ & $76.5 \pm 0.5$ & $82.0 \pm 0.5$ \\
\hline $1-135625$ & 39.33 & $98.0 \pm 1.0$ & $111.0 \pm 2.0$ & $98.0 \pm 1.0$ & $98.0 \pm 1.0$ & $17.5 \pm 1.2$ & $16.0 \pm 1.5$ & $15.0 \pm 0.5$ & $12.5 \pm 1.0$ \\
\hline $1-216958$ & 38.9 & $99.0 \pm 0.0$ & $78.0 \pm 0.0$ & $67.0 \pm 0.0$ & $72.0 \pm 0.0$ & $58.0 \pm 5.8$ & $41.0 \pm 0.5$ & $60.0 \pm 4.5$ & $81.5 \pm 0.5$ \\
\hline $1-135285$ & 38.93 & $133.0 \pm 1.0$ & $155.0 \pm 2.0$ & $166.0 \pm 2.0$ & $162.0 \pm 1.0$ & $116.5 \pm 0.5$ & $112.0 \pm 0.5$ & $114.0 \pm 0.5$ & $113.0 \pm 0.5$ \\
\hline $1-633990$ & 38.77 & $97.0 \pm 0.0$ & $93.0 \pm 1.0$ & $99.0 \pm 0.0$ & $103.0 \pm 1.0$ & $33.5 \pm 1.2$ & $30.0 \pm 0.5$ & $31.5 \pm 0.5$ & $37.5 \pm 0.5$ \\
\hline $1-25688$ & 38.35 & $110.0 \pm 1.0$ & $64.0 \pm 2.0$ & $110.0 \pm 2.0$ & $115.0 \pm 1.0$ & $20.5 \pm 1.5$ & $13.5 \pm 0.5$ & $17.0 \pm 0.8$ & $19.5 \pm 1.5$ \\
\hline
\end{tabular}


Table A2. Parameters of AGN in MaNGA-MPL5: (1) galaxy identification in the MaNGA survey, (2-3) RA/Dec. (2000) in degrees, (4) spectroscopic redshift from SDSS-III, (5) integrated absolute $r$-band magnitude from SDSS-III, (6) stellar mass in units of $M_{\odot}$, errors associated with the stellar masses of galaxies in our sample are typically under 0.03 dex (Conroy, Gunn \& White 2009), (7) elliptical/spiral/merging classification from Galaxy Zoo I, (8-9) $r$-band concentration and asymmetry, and (10) [O III] luminosity in units of $10^{40} \mathrm{erg} \mathrm{s}^{-1}$. Table extracted from Rembold et al. (2017).

\begin{tabular}{|c|c|c|c|c|c|c|c|c|c|}
\hline $\begin{array}{l}\text { mangaid } \\
\text { (1) }\end{array}$ & $\begin{array}{l}\text { RA } \\
(2)\end{array}$ & $\begin{array}{l}\text { Dec. } \\
\text { (3) }\end{array}$ & $\begin{array}{c}z \\
(4)\end{array}$ & $\begin{array}{l}M_{r} \\
(5)\end{array}$ & $\begin{array}{c}\log M^{\star} / M_{\odot} \odot \\
\text { (6) }\end{array}$ & $\begin{array}{c}\mathrm{GZ1}_{c} \\
\text { (7) }\end{array}$ & $\begin{array}{c}C \\
(8)\end{array}$ & $\begin{array}{l}A \\
(9)\end{array}$ & $\begin{array}{c}L([\mathrm{O} \mathrm{III}]) \\
\quad(10)\end{array}$ \\
\hline $1-558912$ & 166.129410 & 42.624554 & 0.1261 & -20.46 & 11.25 & - & 0.37 & 0.12 & $56.82 \pm 1.25$ \\
\hline $1-269632$ & 247.560974 & 26.206474 & 0.1315 & -21.78 & 11.62 & $\mathrm{~S}$ & 0.47 & 0.05 & $30.08 \pm 1.69$ \\
\hline $1-258599$ & 186.181000 & 44.410770 & 0.1256 & -21.24 & 11.68 & $\mathrm{E}$ & 0.50 & 0.11 & $20.95 \pm 0.67$ \\
\hline $1-72322$ & 121.014198 & 40.802612 & 0.1262 & -21.81 & 12.05 & $\mathrm{~S}$ & 0.34 & 0.08 & $20.66 \pm 0.43$ \\
\hline $1-121532$ & 118.091110 & 34.326569 & 0.1400 & -20.51 & 11.34 & $\mathrm{E}$ & 0.33 & 0.05 & $11.68 \pm 0.96$ \\
\hline $1-209980$ & 240.470871 & 45.351940 & 0.0420 & -19.70 & 10.79 & $\mathrm{~S}$ & 0.57 & 0.04 & $11.01 \pm 0.17$ \\
\hline $1-173958$ & 167.306015 & 49.519432 & 0.0724 & -20.53 & 11.31 & $\mathrm{~S}$ & 0.33 & 0.06 & $6.79 \pm 0.30$ \\
\hline $1-338922$ & 114.775749 & 44.402767 & 0.1345 & -20.27 & 11.13 & M & 0.44 & 0.03 & $6.77 \pm 0.90$ \\
\hline $1-279147$ & 168.957733 & 46.319565 & 0.0533 & -19.51 & 10.66 & $\mathrm{~S}$ & 0.45 & 0.03 & $6.77 \pm 0.20$ \\
\hline $1-460812$ & 127.170799 & 17.581400 & 0.0665 & -19.81 & 11.44 & - & 0.38 & 0.05 & $6.46 \pm 0.31$ \\
\hline $1-92866$ & 243.581818 & 50.465611 & 0.0603 & -20.56 & 11.69 & $\mathrm{E}$ & 0.49 & 0.05 & $6.12 \pm 0.30$ \\
\hline $1-94784$ & 249.318420 & 44.418228 & 0.0314 & -20.06 & 10.85 & $\mathrm{~S}$ & 0.42 & 0.03 & $5.96 \pm 0.12$ \\
\hline $1-256446$ & 166.509872 & 43.173473 & 0.0584 & -19.40 & 11.14 & $\mathrm{E}$ & 0.49 & 0.05 & $3.74 \pm 0.15$ \\
\hline $1-95585$ & 255.029877 & 37.839500 & 0.0633 & -20.88 & 11.24 & $\mathrm{~S}$ & 0.27 & 0.08 & $3.58 \pm 0.16$ \\
\hline $1-135641$ & 249.557312 & 40.146820 & 0.0304 & -19.03 & 11.19 & $\mathrm{~S}$ & 0.28 & 0.08 & $3.52 \pm 0.09$ \\
\hline $1-259142$ & 193.703995 & 44.155567 & 0.0543 & -20.75 & 11.29 & $\mathrm{~S}$ & 0.39 & 0.06 & $3.47 \pm 0.20$ \\
\hline $1-109056$ & 39.446587 & 0.405085 & 0.0473 & -19.27 & 10.57 & - & 0.32 & 0.05 & $3.24 \pm 0.08$ \\
\hline $1-24148$ & 258.827423 & 57.658772 & 0.0282 & -18.51 & 10.56 & $\mathrm{~S}$ & 0.31 & 0.04 & $3.17 \pm 0.05$ \\
\hline $1-166919$ & 146.709106 & 43.423843 & 0.0722 & -20.85 & 11.28 & $\mathrm{~S}$ & 0.37 & 0.06 & $2.64 \pm 0.25$ \\
\hline $1-248389$ & 240.658051 & 41.293427 & 0.0348 & -19.36 & 10.57 & $\mathrm{~S}$ & 0.49 & 0.12 & $2.55 \pm 0.09$ \\
\hline $1-321739$ & 226.431656 & 44.404903 & 0.0283 & -18.91 & 11.12 & $\mathrm{~S}$ & 0.40 & 0.14 & $2.24 \pm 0.10$ \\
\hline $1-234618$ & 202.128433 & 47.714039 & 0.0608 & -19.64 & 11.37 & $\mathrm{~S}$ & 0.31 & 0.09 & $2.23 \pm 0.23$ \\
\hline $1-229010$ & 57.243038 & -1.144831 & 0.0407 & -20.51 & 11.46 & - & 0.41 & 0.03 & $2.11 \pm 0.09$ \\
\hline $1-211311$ & 248.426392 & 39.185120 & 0.0298 & -19.04 & 10.44 & $\mathrm{E} / \mathrm{S}$ & 0.43 & 0.02 & $1.99 \pm 0.06$ \\
\hline $1-95092$ & 250.846420 & 39.806461 & 0.0302 & -19.95 & 11.20 & $\mathrm{E}$ & 0.47 & 0.04 & $1.54 \pm 0.07$ \\
\hline $1-279676$ & 173.981888 & 48.021458 & 0.0587 & -19.40 & 10.81 & - & 0.32 & 0.02 & $1.52 \pm 0.14$ \\
\hline $1-201561$ & 118.053215 & 28.772579 & 0.0637 & -19.73 & 10.88 & $\mathrm{~S}$ & 0.30 & 0.07 & $1.37 \pm 0.15$ \\
\hline $1-198182$ & 224.749649 & 48.409855 & 0.0359 & -20.22 & 11.09 & $\mathrm{E}$ & 0.49 & 0.01 & $1.34 \pm 0.11$ \\
\hline $1-96075$ & 253.946381 & 39.310535 & 0.0631 & -21.12 & 11.35 & $\mathrm{~S}$ & 0.29 & 0.07 & $1.26 \pm 0.13$ \\
\hline $1-519742$ & 206.612457 & 22.076742 & 0.0276 & -17.62 & 9.64 & $\mathrm{~S}$ & 0.23 & 0.04 & $1.19 \pm 0.03$ \\
\hline $1-491229$ & 172.607544 & 22.216530 & 0.0393 & -20.25 & 11.12 & $\mathrm{E}$ & 0.51 & 0.02 & $1.14 \pm 0.11$ \\
\hline $1-604761$ & 113.472275 & 37.025906 & 0.0618 & -20.92 & 11.34 & $\mathrm{~S}$ & 0.26 & 0.12 & $1.00 \pm 0.13$ \\
\hline $1-25725$ & 262.996735 & 59.971638 & 0.0291 & -18.30 & 10.55 & $\mathrm{E}$ & 0.44 & 0.04 & $0.92 \pm 0.05$ \\
\hline $1-94604$ & 251.335938 & 42.757790 & 0.0493 & -19.44 & 10.52 & $\mathrm{~S}$ & 0.37 & 0.01 & $0.86 \pm 0.07$ \\
\hline $1-37036$ & 41.699909 & 0.421577 & 0.0283 & -19.02 & 10.66 & $\mathrm{E}$ & 0.40 & 0.09 & $0.84 \pm 0.06$ \\
\hline $1-167688$ & 155.885559 & 46.057755 & 0.0258 & -17.86 & 9.75 & $\mathrm{E}$ & 0.52 & 0.04 & $0.84 \pm 0.02$ \\
\hline $1-279666$ & 173.911240 & 47.515518 & 0.0455 & -18.83 & 10.42 & $\mathrm{E}$ & 0.31 & 0.02 & $0.84 \pm 0.07$ \\
\hline $1-339163$ & 116.280205 & 46.072422 & 0.0312 & -20.02 & 10.97 & $\mathrm{~S}$ & 0.30 & 0.10 & $0.82 \pm 0.07$ \\
\hline $1-258774$ & 186.400864 & 45.083858 & 0.0384 & -19.60 & 10.77 & - & 0.55 & 0.03 & $0.77 \pm 0.10$ \\
\hline $1-198153$ & 224.289078 & 48.633968 & 0.0354 & -19.83 & 11.00 & $\mathrm{~S}$ & 0.27 & 0.07 & $0.76 \pm 0.08$ \\
\hline $1-91016$ & 234.810974 & 56.670856 & 0.0463 & -18.60 & 10.56 & $\mathrm{~S}$ & 0.27 & 0.06 & $0.76 \pm 0.09$ \\
\hline $1-279073$ & 170.588150 & 46.430504 & 0.0323 & -19.53 & 10.79 & $\mathrm{E}$ & 0.51 & 0.01 & $0.63 \pm 0.06$ \\
\hline $1-135044$ & 247.907990 & 41.493645 & 0.0303 & -19.76 & 10.65 & $\mathrm{~S}$ & 0.31 & 0.05 & $0.61 \pm 0.04$ \\
\hline
\end{tabular}


Table A2 - continued

\begin{tabular}{|c|c|c|c|c|c|c|c|c|c|}
\hline $\begin{array}{l}\text { mangaid } \\
\text { (1) }\end{array}$ & $\begin{array}{l}\text { RA } \\
(2)\end{array}$ & $\begin{array}{l}\text { Dec. } \\
(3)\end{array}$ & $\begin{array}{c}z \\
(4)\end{array}$ & $\begin{array}{l}M_{r} \\
(5)\end{array}$ & $\begin{array}{c}\log M^{\star} / M_{\odot} \\
\text { (6) }\end{array}$ & $\begin{array}{c}\mathrm{GZ1} 1_{c} \\
(7)\end{array}$ & $\begin{array}{c}C \\
(8)\end{array}$ & $\begin{array}{c}A \\
(9)\end{array}$ & $\begin{array}{l}L([\mathrm{O} \text { III }]) \\
\quad(10)\end{array}$ \\
\hline $1-148068$ & 156.805679 & 48.244793 & 0.0610 & -20.72 & 11.41 & $\mathrm{~S}$ & 0.22 & 0.04 & $0.45 \pm 0.15$ \\
\hline $1-277552$ & 167.034561 & 45.984623 & 0.0362 & -19.72 & 10.83 & $\mathrm{~S}$ & 0.21 & 0.15 & $0.44 \pm 0.05$ \\
\hline $1-217050$ & 136.719986 & 41.408253 & 0.0274 & -19.66 & 10.93 & $\mathrm{E}$ & 0.47 & 0.02 & $0.43 \pm 0.03$ \\
\hline $1-25554$ & 262.486053 & 58.397408 & 0.0268 & -19.27 & 10.52 & $\mathrm{~S}$ & 0.36 & 0.04 & $0.24 \pm 0.03$ \\
\hline $1-135285$ & 247.216949 & 42.812012 & 0.0316 & -19.66 & 10.78 & - & 0.32 & 0.05 & $0.20 \pm 0.04$ \\
\hline
\end{tabular}

Table A3. Control sample parameters: (1) identification of the AGN host associated with the control galaxy and (2-11) same as (1-10) of Table A2. A total of 12 control sample objects have been paired to two different AGN hosts and appear more than once in the table. Table extracted from Rembold et al. (2017).

\begin{tabular}{|c|c|c|c|c|c|c|c|c|c|c|}
\hline $\begin{array}{l}\text { AGN mangaid } \\
\text { (1) }\end{array}$ & $\begin{array}{l}\text { mangaid } \\
\text { (2) }\end{array}$ & $\begin{array}{l}\text { RA } \\
\text { (3) }\end{array}$ & $\begin{array}{l}\text { Dec. } \\
(4)\end{array}$ & $\begin{array}{c}z \\
(5)\end{array}$ & $\begin{array}{l}M_{r} \\
\text { (6) }\end{array}$ & $\begin{array}{c}\log M^{\star} / M_{\odot} \\
\text { (7) }\end{array}$ & $\begin{array}{l}\mathrm{GZ1}_{c} \\
(8)\end{array}$ & $\begin{array}{c}C \\
(9)\end{array}$ & $\begin{array}{c}A \\
(10)\end{array}$ & $\begin{array}{l}L([\mathrm{O} I I I]) \\
\quad(11)\end{array}$ \\
\hline \multirow[t]{2}{*}{$1-558912$} & $1-71481$ & 117.456001 & 34.883911 & 0.1312 & -20.95 & 11.70 & E & 0.47 & 0.02 & $0.10 \pm 0.20$ \\
\hline & $1-72928$ & 127.256485 & 45.016773 & 0.1270 & -20.62 & 11.52 & E & 0.40 & 0.21 & $0.09 \pm 0.23$ \\
\hline \multirow[t]{2}{*}{$1-269632$} & $1-210700$ & 248.140564 & 39.131020 & 0.1303 & -20.96 & 11.67 & S & 0.36 & 0.03 & $1.55 \pm 0.44$ \\
\hline & $1-378795$ & 118.925613 & 50.172771 & 0.0967 & -20.77 & 11.35 & S & 0.32 & 0.03 & $0.72 \pm 0.31$ \\
\hline \multirow[t]{2}{*}{$1-258599$} & $1-93876$ & 246.942947 & 44.177521 & 0.1394 & -20.75 & 11.50 & E & 0.44 & 0.01 & $0.46 \pm 0.36$ \\
\hline & $1-166691$ & 146.047348 & 42.900040 & 0.1052 & -20.50 & 11.36 & E & 0.51 & 0.04 & $0.09 \pm 0.49$ \\
\hline \multirow[t]{2}{*}{$1-72322$} & $1-121717$ & 118.803429 & 35.596798 & 0.1098 & -21.11 & 11.61 & S & 0.39 & 0.12 & $1.40 \pm 0.57$ \\
\hline & $1-43721$ & 116.967567 & 43.383499 & 0.1114 & -21.41 & 11.86 & S & 0.32 & 0.01 & $1.91 \pm 0.52$ \\
\hline \multirow{2}{*}{$1-121532$} & $1-218427$ & 124.342316 & 27.796206 & 0.1496 & -21.30 & 11.47 & E & 0.47 & 0.04 & $0.72 \pm 0.62$ \\
\hline & $1-177493$ & 257.085754 & 31.746916 & 0.1081 & -20.90 & 11.30 & E & 0.38 & 0.06 & $2.29 \pm 0.28$ \\
\hline \multirow[t]{2}{*}{$1-209980$} & $1-295095$ & 248.348663 & 24.776577 & 0.0410 & -18.40 & 10.14 & E & 0.35 & 0.05 & $0.15 \pm 0.03$ \\
\hline & $1-92626$ & 241.799545 & 48.572563 & 0.0434 & -20.04 & 11.04 & S & 0.36 & 0.03 & $0.76 \pm 0.07$ \\
\hline \multirow[t]{2}{*}{$1-44379$} & $1-211082$ & 247.620041 & 39.626045 & 0.0304 & -19.72 & 11.07 & E & 0.31 & 0.06 & $0.19 \pm 0.04$ \\
\hline & $1-135371$ & 250.156235 & 39.221634 & 0.0352 & -19.20 & 10.76 & S & 0.28 & 0.11 & $0.25 \pm 0.07$ \\
\hline \multirow[t]{2}{*}{$1-149211$} & $1-377321$ & 110.556152 & 42.183643 & 0.0444 & -19.02 & 9.89 & S & 0.31 & 0.03 & $4.53 \pm 0.13$ \\
\hline & $1-491233$ & 172.563995 & 22.992010 & 0.0332 & -18.39 & 10.59 & S & 0.29 & 0.06 & $0.25 \pm 0.03$ \\
\hline \multirow[t]{2}{*}{ 1-173958 } & $1-247456$ & 232.823196 & 45.416538 & 0.0705 & -20.05 & 10.83 & - & 0.40 & 0.02 & $0.57 \pm 0.16$ \\
\hline & $1-24246$ & 264.840790 & 56.567070 & 0.0818 & -19.91 & 10.57 & S & 0.75 & 0.36 & $0.11 \pm 0.06$ \\
\hline \multirow[t]{2}{*}{$1-338922$} & $1-286804$ & 211.904861 & 44.482269 & 0.1429 & -20.03 & 10.50 & M & 0.44 & 0.32 & $2.23 \pm 0.43$ \\
\hline & $1-109493$ & 56.425140 & -0.378460 & 0.1093 & -20.46 & 11.26 & - & 0.49 & -0.01 & $0.15 \pm 0.18$ \\
\hline \multirow[t]{2}{*}{$1-279147$} & $1-283246$ & 191.078873 & 46.407131 & 0.0496 & -19.17 & 10.55 & S & 0.47 & 0.04 & $0.23 \pm 0.06$ \\
\hline & $1-351538$ & 119.145126 & 47.563850 & 0.0692 & -19.67 & 11.00 & S & 0.35 & 0.08 & $0.46 \pm 0.13$ \\
\hline \multirow{2}{*}{$1-460812$} & $1-270160$ & 248.274612 & 26.211815 & 0.0660 & -20.37 & 11.46 & S & 0.50 & 0.02 & $0.70 \pm 0.39$ \\
\hline & $1-258455$ & 183.612198 & 45.195454 & 0.0653 & -20.02 & 11.03 & E & 0.40 & 0.03 & $0.49 \pm 0.14$ \\
\hline \multirow[t]{2}{*}{$1-92866$} & $1-94514$ & 248.241180 & 42.524670 & 0.0614 & -20.60 & 11.17 & E & 0.51 & 0.00 & - \\
\hline & $1-210614$ & 244.501755 & 41.392189 & 0.0612 & -20.64 & 11.48 & E & 0.49 & 0.01 & $0.40 \pm 0.14$ \\
\hline \multirow[t]{2}{*}{$1-94784$} & $1-211063$ & 247.058411 & 40.313835 & 0.0331 & -19.87 & 10.79 & S & 0.33 & 0.09 & $0.20 \pm 0.04$ \\
\hline & $1-135502$ & 247.764175 & 39.838505 & 0.0305 & -19.51 & 11.13 & S & 0.40 & 0.09 & $0.50 \pm 0.05$ \\
\hline \multirow[t]{2}{*}{$1-44303$} & $1-339028$ & 116.097923 & 44.527740 & 0.0497 & -20.01 & 11.24 & $S$ & 0.36 & 0.06 & $0.44 \pm 0.08$ \\
\hline & $1-379087$ & 119.910118 & 51.792362 & 0.0534 & -19.60 & 11.02 & S & 0.38 & 0.10 & $0.72 \pm 0.13$ \\
\hline \multirow[t]{2}{*}{$1-339094$} & $1-274646$ & 158.017029 & 43.859268 & 0.0284 & -18.70 & 10.36 & E & 0.53 & 0.02 & $0.35 \pm 0.04$ \\
\hline & $1-24099$ & 258.027618 & 57.504009 & 0.0282 & -18.67 & 10.34 & E & 0.44 & 0.01 & $0.06 \pm 0.03$ \\
\hline \multirow[t]{2}{*}{$1-137883$} & $1-178838$ & 312.023621 & 0.068841 & 0.0247 & -17.54 & 10.46 & - & 0.51 & 0.19 & $0.10 \pm 0.02$ \\
\hline & $1-36878$ & 42.542126 & -0.867116 & 0.0232 & -18.88 & 10.77 & E & 0.45 & 0.07 & $0.28 \pm 0.04$ \\
\hline \multirow[t]{2}{*}{$1-48116$} & $1-386452$ & 136.228333 & 28.384314 & 0.0269 & -19.54 & 10.57 & S & 0.49 & 0.09 & $0.32 \pm 0.04$ \\
\hline & $1-24416$ & 263.033173 & 56.878746 & 0.0281 & -19.16 & 10.66 & S & 0.37 & 0.03 & $0.22 \pm 0.03$ \\
\hline \multirow[t]{2}{*}{$1-256446$} & $1-322671$ & 235.797028 & 39.238773 & 0.0637 & -19.77 & 10.82 & E & 0.49 & 0.04 & - \\
\hline & $1-256465$ & 166.752243 & 43.089901 & 0.0575 & -19.70 & 10.79 & E & 0.50 & 0.01 & $0.59 \pm 0.11$ \\
\hline \multirow[t]{2}{*}{$1-95585$} & $1-166947$ & 147.335007 & 43.442989 & 0.0720 & -20.79 & 10.81 & S & 0.29 & 0.02 & $0.13 \pm 0.08$ \\
\hline & $1-210593$ & 244.419754 & 41.899155 & 0.0605 & -19.76 & 10.90 & S & 0.36 & 0.06 & $0.43 \pm 0.14$ \\
\hline $1-135641$ & $1-635503$ & 318.990448 & 9.543076 & 0.0293 & -19.37 & 10.91 & S & 0.22 & 0.10 & $0.15 \pm 0.06$ \\
\hline & $1-235398$ & 213.149185 & 47.253059 & 0.0281 & -18.91 & 10.99 & S & 0.28 & 0.10 & $0.16 \pm 0.05$ \\
\hline $1-259142$ & $1-55572$ & 133.121307 & 56.112690 & 0.0454 & -20.11 & 11.03 & S & 0.40 & 0.06 & $0.12 \pm 0.04$ \\
\hline & $1-489649$ & 171.954834 & 21.386103 & 0.0406 & -19.94 & 10.95 & S & 0.40 & 0.03 & $0.30 \pm 0.08$ \\
\hline $1-109056$ & $1-73005$ & 125.402306 & 45.585476 & 0.0514 & -19.47 & 10.65 & S & 0.31 & 0.05 & $0.20 \pm 0.06$ \\
\hline & $1-43009$ & 113.553879 & 39.076836 & 0.0510 & -19.41 & 10.43 & S & 0.26 & 0.03 & $0.12 \pm 0.04$ \\
\hline
\end{tabular}


Table A3 - continued

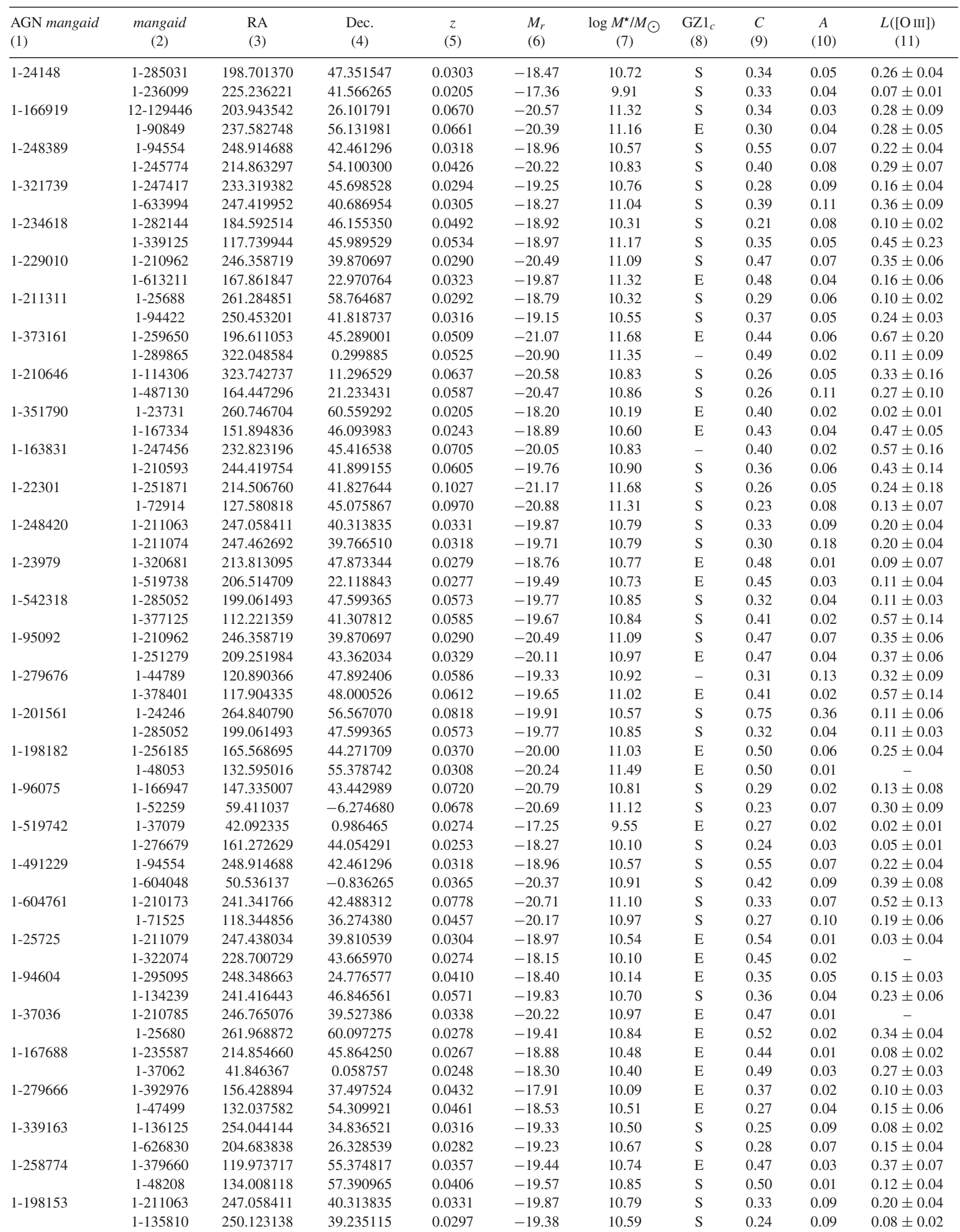


Table A3 - continued

\begin{tabular}{lcccccccccc}
\hline $\begin{array}{l}\text { AGN mangaid } \\
(1)\end{array}$ & $\begin{array}{c}\text { mangaid } \\
(2)\end{array}$ & $\begin{array}{c}\text { RA } \\
(3)\end{array}$ & $\begin{array}{c}\text { Dec. } \\
(4)\end{array}$ & $\begin{array}{c}z \\
(5)\end{array}$ & $\begin{array}{c}M_{r} \\
(6)\end{array}$ & $\begin{array}{c}\log M^{\star} / M_{\odot} \\
(7)\end{array}$ & $\begin{array}{c}\mathrm{GZ1} 1_{c} \\
(8)\end{array}$ & $\begin{array}{c}C \\
(9)\end{array}$ & $\begin{array}{c}A \\
(10)\end{array}$ & $\begin{array}{c}L([\mathrm{O} \text { III }]) \\
(11)\end{array}$ \\
\hline $1-91016$ & $1-338828$ & 115.641609 & 44.215858 & 0.0418 & -18.10 & 10.42 & $\mathrm{~S}$ & 0.28 & 0.03 & $0.43 \pm 0.05$ \\
& $1-386695$ & 137.983505 & 27.899269 & 0.0474 & -19.33 & 10.48 & $\mathrm{~S}$ & 0.27 & 0.09 & $0.81 \pm 0.09$ \\
$1-279073$ & $1-211100$ & 247.830322 & 39.744129 & 0.0309 & -19.15 & 10.62 & $\mathrm{E}$ & 0.56 & 0.02 & - \\
& $1-210784$ & 247.097122 & 39.570305 & 0.0292 & -19.61 & 10.86 & $\mathrm{E}$ & 0.48 & 0.00 & $0.15 \pm 0.05$ \\
$1-135044$ & $1-218280$ & 124.003311 & 27.075895 & 0.0255 & -19.57 & 10.81 & $\mathrm{~S}$ & 0.27 & 0.08 & $0.12 \pm 0.03$ \\
& $1-211063$ & 247.058411 & 40.313835 & 0.0331 & -19.87 & 10.79 & $\mathrm{~S}$ & 0.33 & 0.09 & $0.20 \pm 0.04$ \\
$1-148068$ & $1-166947$ & 147.335007 & 43.442989 & 0.0720 & -20.79 & 10.81 & $\mathrm{~S}$ & 0.29 & 0.02 & $0.13 \pm 0.08$ \\
& $1-55572$ & 133.121307 & 56.112690 & 0.0454 & -20.11 & 11.03 & $\mathrm{~S}$ & 0.40 & 0.06 & $0.12 \pm 0.04$ \\
$1-277552$ & $1-264513$ & 236.941513 & 28.641697 & 0.0333 & -20.92 & 11.28 & $\mathrm{~S}$ & 0.25 & 0.18 & $0.33 \pm 0.05$ \\
& $1-136125$ & 254.044144 & 34.836521 & 0.0316 & -19.33 & 10.50 & $\mathrm{~S}$ & 0.25 & 0.09 & $0.08 \pm 0.02$ \\
$1-217050$ & $1-135372$ & 250.116714 & 39.320118 & 0.0301 & -20.29 & 11.08 & $\mathrm{E}$ & 0.49 & 0.02 & $0.01 \pm 0.23$ \\
& $1-274663$ & 157.660522 & 44.012722 & 0.0280 & -19.88 & 11.00 & $\mathrm{E}$ & 0.50 & 0.01 & $0.08 \pm 0.02$ \\
$1-25554$ & $1-135625$ & 248.507462 & 41.347946 & 0.0284 & -19.06 & 10.56 & $\mathrm{~S}$ & 0.43 & 0.05 & $0.56 \pm 0.04$ \\
& $1-216958$ & 136.200287 & 40.591721 & 0.0270 & -18.95 & 10.41 & $\mathrm{~S}$ & 0.51 & 0.03 & $0.23 \pm 0.02$ \\
$1-135285$ & $1-633990$ & 247.304123 & 41.150871 & 0.0296 & -19.06 & 10.46 & $\mathrm{~S}$ & 0.34 & 0.03 & $0.25 \pm 0.03$ \\
& $1-25688$ & 261.284851 & 58.764687 & 0.0292 & -18.79 & 10.32 & $\mathrm{~S}$ & 0.29 & 0.06 & $0.10 \pm 0.02$ \\
\hline
\end{tabular}

This paper has been typeset from a $\mathrm{T}_{\mathrm{E}} \mathrm{X} / \mathrm{L} \mathrm{T}_{\mathrm{E}} \mathrm{X}$ file prepared by the author. 